\title{
WGCNA Co-Expression Network Analysis Reveals LncRNA LINC00313 Functions as A ceRNA to Regulate PTEN-Mediated PI3K/AKT Signaling Pathway by Sponging miR-19a-3p in Docetaxel- Resistant Prostate Cancer Cells
}

\section{Bin shen Chen}

Zhujiang Hospital

Kai Xu

Zhujiang Hospital

Yiming Zhang

Zhujiang Hospital

\section{Peng Xu}

Zhujiang Hospital

Chaoming Li

Zhujiang Hospital

Jun Liu

Zhujiang Hospital

Yawen Xu ( $\sim$ yawenxu72@163.com)

Zhujiang Hospital, Southern Medical University

\section{Research}

Keywords: LINC00313, docetaxel resistance, proliferation, metastasis, prostate cancer, PI3K/Akt

Posted Date: October 21st, 2020

DOI: https://doi.org/10.21203/rs.3.rs-94032/v1

License: (c) (i) This work is licensed under a Creative Commons Attribution 4.0 International License.

Read Full License 


\section{Abstract}

Background: Docetaxel is the preferred chemotherapeutic agent for patients with hormone-refractory PCa. LncRNAs play important roles in the development of docetaxel resistance in patients with PCa. We evaluated the effects of the IncRNA LINC00313 on growth and metastasis in docetaxel-resistant PCa cells, and the molecular mechanisms underlying these effects.

Methods: The mRNA dataset (GSE102124 and GSE55945) and IncRNA dataset (GSE115414) were downloaded from the GEO database. The DEGs were identified using the "R package limma". WGCNA were performed to identify the modules and genes associated with PCa. A PPI network was constructed using the STRING database and visualized using Cytoscape. The Cytohubba plug-in was used to identify the hub genes and cytoNCA plug-in was used to identify the highest linkage hub genes. Based on IncRNAmiRNA pairs and miRNA-mRNA pairs using StarBase and TargetScan databases, a ceRNA network was constructed. Functional analysis was performed via GO, GSEA, and GSVA analysis. Cell proliferation, apoptosis, cell cycle progression, migration, and invasion were evaluated by CCK-8, colony formation, flow cytometry, transwell, and wound healing assays. RT-PCR, western blot, and immunohistochemistry were used to evaluate mRNA and protein expression. The oncogenicity of LINC00313 in docetaxelresistant PCa cells was determined by tumor transplantation in nude mice. Luciferase reporter and RNA pull-down assays were used to evaluate interactions between LINC00313 and miR-19a-3p as well as between miR-19a-3p and PTEN.

Results: LINC00313-miR-19a-3p-PTEN was a core network of ceRNA network. Cox regression analysis identified LINC00313 as an independent prognostic variable for OS in patients with PCa. LINC00313 overexpression in docetaxel-resistant PCa cells suppressed proliferation, invasion, and migration, and induced apoptosis and cell cycle arrested at the G1 phase. These changes were related to changes in cyclinD1, p27, Ki67, Bax, Bcl-2, caspase-3/9, E-cadherin, N-cadherin, Vimentin, and MMP-2 expression. Animal experiments showed that LINC00313 silencing promoted tumor formation. Co-transfection with LINC00313-WT and miR-19a-3p mimic reduced luciferase activity, and co-transfection with PTEN-WT and LINC00313 increased luciferase activity. The effects of LINC00313, including increased PTEN, decreased PIP3 expression, and the inhibition of proliferation and metastasis in docetaxel-resistant PC cells, were abolished by miR-19a-3p mimic.

Conclusion: LINC00313 overexpression suppressed the proliferation and metastasis of docetaxelresistant PC cells by competitively binding to miR-19a-3p to regulate PTEN expression and inhibit the activation of the PI3K/Akt signaling pathway.

\section{Introduction}

$\mathrm{PCa}$ is a serious health issue, particularly in elderly men. The incidence of PCa in China is increasing rapidly, and many patients exhibit distant invasion and metastasis [1]. Standard endocrine castration therapy (androgen deprivation therapy) is the first-line treatment [2]. However, most patients eventually 
progress to CRPC. Various mechanisms may explain this progression, including (1) abnormal expression of apoptosis-related genes, oncogenes, tumor suppressor genes, and signaling pathways; (2) presence of tumor stem cells; (3) androgen receptor variation; (4) androgen receptor hyperactivation; and (5) ligandindependent androgen receptor activation [3-5]. However, the specific mechanism is still unclear.

CRPC remains a challenge with respect to clinical treatment. Paclitaxel [6], docetaxel [7], and cabazitaxel [8] are used as first-line chemotherapy and androgen receptor-targeted drugs (enzalutamide and abiraterone) are used as second-line drugs. However, about $1 \%$ of the patients are insensitive to these therapies. On average, about 24 months after the development of drug resistance (primary resistance), PCa worsens, and acquired resistance eventually develops [9]. Therefore, detailed analyses of the mechanism underlying the development of drug resistance in PCa, and the identification of key cellular signal transduction pathways are urgently needed for the development of a new therapeutic strategy.

LncRNAs are a class of untranslated transcripts that are more than $200 \mathrm{bp}$ in length. They are widely found in eukaryotic cells and can affect tumor cell proliferation, growth, and angiogenesis via transcriptional activation and interference, genomic imprinting, X-chromosome silencing, regulation of proto-oncogene activation, chromatin modification, and intranuclear transport [10-17]. LncRNAs contribute to the occurrence and progression of PCa by regulating the cell cycle, Toll-like receptors, epithelial-mesenchymal transformation, and drug resistance development $[18,19]$. LINC00313 is overexpressed in thyroid carcinoma and its downregulation inhibits the proliferation, migration, and invasion of thyroid cancer cells by inhibiting the activation of the Akt/mTOR signaling pathway [20]. LINC00313 acts as an oncogene in papillary thyroid cancer by sponging miR-4429 and is recognized as a differentially expressed IncRNA in early-stage lung cancer [21, 22]. These previous studies clearly establish the relationship between LINC00313 and cancer. Furthermore, studies have shown that LINC00313 is a member of the IncRNA-miRNA-mRNA network in PC [23]. However, detailed analyses of LINC00313 have focused on thyroid cancer and papillary thyroid cancer, and little is known about its roles in other cancers. An analysis based on GEO database has shown that LINC00313 is a differentially expressed IncRNA in PCa, and downregulation in tumor tissues. Therefore, in this study, the roles and mechanism of action of LINC00313 were comprehensively evaluated in PCa.

\section{Materials And Methods}

\section{Microarray data and Differential expression analysis}

The microarray data of a IncRNA expression profiles were collected from the GEO database (GSE115414) and the microarray data of two mRNA expression profiles (GSE55945 and GSE102124) were collected from the GEO database. We used the Limma R package to explore the significantly differentially expressed genes between tumorous and normal tissues. The cut-off value was $\| \log 2 \mathrm{FC} \mid>1$ and FDR $<0.05$ (FC, fold change; FDR, false discovery rate). Due to the public availability of data on the GEO database, ethical approval or informed consent was not required for this study.

\section{Ethics statement}


Approval of this study protocol was obtained from the Ethics Committee of Zhujiang Hospital, Southern Medical University, and written informed consent was provided from all subjects. All experiments were performed in accordance with the relevant guidelines and regulations of Zhujiang Hospital, Southern Medical University. This study conformed to the principles outlined in the Declaration of Helsinki adopted by the World Medical Association's General Assembly.

\section{Predictions of target miRNA and miRNA target genes}

The Starbase and miRbase databases were used to predict target miRNAs, their miRNA binding sites and the structure of miRNA. Interactions between miRNA and mRNA were predicted based on the Starbase database. Moreover, intersections with the DEGs were identified to screen out the hub genes targeted by the potential target miRNAs. Only one target mRNA recognized was considered as candidate mRNAs.

\section{Construction of the CeRNA network}

The IncRNA-miRNA-mRNA regulatory network was constructed by using a combination of IncRNA-miRNA pairs and miRNA-mRNA pairs and visualized by Cytoscape 3.6.1.

\section{Prediction of target miRNA and mRNA}

StarBase V2.0 database (http://starbase.sysu.edu.cn/starbase2/index.php) is used to predict the target miRNAs of LINC00313 and TargetScan 7.2 (http://www.targetscan.org/vert_72/) is used to predict the target mRNAs of miR-19a-3p.

\section{GO functional analysis}

The DEGs were evaluated by GO annotation using clusterProfiler package in R. P value $<0.05$ served as the cut-off point to assess the functional pathways.

\section{Gene functional analysis}

GSEA is a computational method for assessing whether a set of genes defined by a priori show statistically significant, consistent differences between two biological states. GSEA was performed to analyze the enrichment of datasets between control group and experiment group. A false discovery rate (FDR) $<25 \%$ and nominal $P$-value $<5 \%$ were set as the cut-off criteria. In addition, GSVA was used to find the most relevant pathways of hub genes, according to the gene sets files from the KEGG databases.

\section{PPI network construction and hub genes selection}

The STRING database (http://string-db.org/) provides a significant association of protein-protein interactions (PPIs). Cytoscape is used for the visual exploration of interaction networks. In this study, DEG PPI networks were analyzed by the STRING database and subsequently visualized by using Cytoscape. The cutoff criterion was set as a combined score $>0.4$. The plug-in CytoHubba of Cytoscape was used to 
identify the top 10 hub genes according to 'Degree' method. In addition, other add-ins of Cytoscape, CytoNCA, was employed to discover the highest linkage hub genes in the network

\section{Patients and tissue samples}

A total of 82 prostate cancer patients admitted to Zhujiang Hospital, Southern Medical University between 2015 and 2018, were included in this study. All patients had undergone a radical prostatectomy, and had not received chemotherapy, radiation therapy or androgen-deprivation treatment. Prostate cancer tissue samples were placed into $10 \%$ buffered formalin, and then subjected to gradient dehydration, wax dipping and embedding. Sections (5- $\mu \mathrm{m}$ thick) of each sample were placed on glass slides, dewaxed, dehydrated and stained with hematoxylin-eosin. All paraffin-embedded tissues for each sample were subject to histopathological examination by hematoxylin-eosin staining. The histopathological grading of samples for all cases was performed by experienced pathologists on hematoxylin-eosin stained sections to confirm diagnosis and tumor content as $>70 \%$ prostate cancer cells in the tissue samples. The clinicopathological and demographic data pre- and post-operation were preserved in medical records. The biochemical and clinicopathological parameters for each patient, including clinical stage, Gleason score, margin status, angiolymphatic invasion status, seminal vesicle invasion status and biochemical recurrence were all recorded. The summary of clinicopathological characteristics for all patients is presented in Table 1. The biochemical recurrence (BCR) is a surrogate endpoint when prostate-specific antigen (PSA) levels are $\geq 0.2 \mathrm{ng} / \mathrm{ml}$ in the patient's serum following surgical treatment. The date of prostatectomy was recognized as the beginning of the follow-up period. Any patients that did not survive due to unexpected events or diseases other than prostate cancer were excluded from the study. The primary prostate cancer tissues and paired noncancerous prostate tissues from 82 cases were collected and frozen in liquid nitrogen and stored at $-80^{\circ} \mathrm{C}$ prior to use.

\section{Cell Culture}

Human prostate cell lines, LNcap, PC3 and DU145 were originally obtained from American Type Culture Collection (ATCC) (Manassas, VA, USA). Cells were maintained in RPMI 1640 medium (Gibco, Rockville, MD, USA) supplemented with $10 \%$ fetal bovine serum (Sigma Aldrich, Oakville, ON) and $100 \mathrm{U} / \mathrm{ml}$ of penicillin/streptomycin (Life Technologies, Burlington, $\mathrm{ON}$ ) at $37^{\circ} \mathrm{C}$ in a humidified atmosphere with $5 \%$ $\mathrm{CO}_{2}$. Docetaxel was purchased from Sigma-Aldrich (Sigma-Aldrich, St. Louis, MO).

\section{Establishment of Dox-resistant cells}

Dox-resistant cells PC3-DR and DU145-DR were incubated from parental PC3 and DU145 cells under continuous exposure to Dox with gradually increasing concentrations of docetaxel from $5 \mathrm{nM}$ to $200 \mathrm{nM}$ in the culture medium. Briefly, PC3 and DU145 cells were incubated first with $5 \mathrm{nM}$ Dox for 2 days followed by incubation in the absence of Dox until the Dox-sensitive clones died. Then, survivable cells were cultured in the presence of $10 \mathrm{nM}$ Dox. The same procedure was repeated until cells that were viable at $200 \mathrm{nM}$ Dox were obtained. The surviving cells repopulated and after 10 months, the cells dividing 
freely in $10 \mathrm{nM}$ and $200 \mathrm{nM}$ Dox-containing media were considered as resistant cell lines and labeled as "PC3-DR" and "DU145-DR," respectively.

\section{Cell transfections}

Vectors expressing LINC00313 and PTEN were constructed by GenePharma (Shanghai, China). Empty vectors were also purchased from GenePharma. Small interfering (si) RNA targeting LINC00313\#1 or LINC00313\#2 and siPTEN and nontargeting negative control (siNC) were obtained from GenePharma (Shanghai, China). MiR-19a-3p mimic, miR-19a-3p inhibitor and scrambled negative control miRNA (miR Ctrl mimic/inhibitor) were purchased from Sigma-Aldrich. Cell transfections were performed using Lipofectamine 2000 Reagent (Invitrogen, Carlsbad, CA) with all operations performed in strict accordance with manufacturer's instructions. Cells were transfected with $15 \mathrm{nM}$ vectors, $20 \mathrm{nM}$ siRNAs and $40 \mathrm{nM}$ miRNAs, respectively, when cells reached $30-50 \%$ confluence. After 48 hours of transfection using Lipofectamine 2000 (Life Technologies) according to the manufacturer's protocol.

\section{Flow cytometry analysis for cell cycle}

Flow cytometry was applied to evaluate cell cycle progression. Briefly, $2 \times 10^{5}$ cells were placed into 6well plates and incubated for 48 hours. Subsequently, cells were collected, washed three times with icecold PBS, fixed with ice-cold $70 \%$ ethanol overnight and stained using propidium iodide. Finally, cell cycle was evaluated using the FACSVerse flow cytometer (Becton Dickinson). All the experiments were performed in triplicates.

\section{Western blot assays}

Proteins were extracted from clinical tissue samples and culturing cells using the RIPA buffer supplemented with proteinase inhibitor cocktail (Pierce Biotechnology, IL, USA) according to the manufacturer's protocols. Protein concentrations were determined using the BCA Protein Assay Kit (Pierce). Extracted proteins were isolated using 10\% SDS-polyacrylamide gel electrophoresis and then transferred onto the PVDF membranes, followed by $5 \%$ nonfat milk blocking. Afterward, the membranes were probed using primary antibodies. The primary antibodies were listed as followed: anti-cyclinD1, antip27, anti-Ki67, anti-Bax, anti-Bcl-2, anti-cleaved caspase-3/-9, anti-E-cadherin, anti-N-cadherin, antiVimentin, anti-MMP2, anti-GAPDH, anti-PTEN, anti-PIP3, anti-PI3K, anti-AKT, anti- phosphorylation (phosph)-PI3K, anti-phosph-AKT, and anti-GAPDHFollowing incubation with HRP-conjugated secondary antibody, the blots were visualized using the ECL detection system (Amersham, Shanghai, China).

\section{RT-PCR analysis}

Total RNAs were collected from the patient specimens and cultured cells using TRIzol reagent (Thermo Fisher Scientific, Inc.) according to the manufacturer's protocol. RNA $(2 \mu \mathrm{g})$ was reversed transcribed into cDNA using TaqMan ${ }^{T M}$ Reverse Transcription reagents (Thermo Fisher Scientific, Inc.). The expression levels of miRNA-19a-3p, PTEN, and LINC00313 were analyzed using the DyNAmo ColorFlash SYBR-Green 
qPCR kit (Thermo Fisher Scientific, Inc.) with the ABI Prism 7700 Sequence Detection system (Applied Biosystems; Thermo Fisher Scientific, Inc.). U6 and GAPDH were used as the internal controls. The sequences of the primers were listed in Table 2. The reaction conditions were: $95^{\circ} \mathrm{C}$ for $30 \mathrm{sec}$, followed by 40 cycles of $95^{\circ} \mathrm{C}$ for $3 \mathrm{sec}$ and $60^{\circ} \mathrm{C}$ for $34 \mathrm{sec}$. The relative expression of target genes was calculated using the $2^{-\triangle \triangle C q}$ method.

\section{Cell viability assay}

After transfection, CCK-8 assay was performed to detect cell proliferation. Cells were collected and cell suspensions with a cell density of $6 \times 10^{4}$ cells per $\mathrm{ml}$ were prepared. Each well of a 96 -well plate was filled with a $0.1-\mathrm{mL}$ cell suspension containing $6 \times 10^{3}$ cells and cells were cultured in an incubator $\left(37^{\circ} \mathrm{C}\right.$, $5 \% \mathrm{CO}_{2}$ ). CCK-8 solution (10 uL, Sigma-Aldrich) was added $24,48,72$, and $96 \mathrm{~h}$ later. After that, cells were cultured for an additional $4 \mathrm{~h}$ and $\mathrm{OD}$ values at $450 \mathrm{nM}$ were measured using a Fisherbrand ${ }^{\mathrm{TM}}$ accuSkan $^{\text {TM }}$ GO UV/Vis Microplate Spectrophotometer (Fisher Scientific).

\section{Cell apoptosis assay}

After transfection, cell apoptosis assay was performed to detect cell apoptosis. Cells were mixed with serum-free medium to prepare cell suspensions at a cell density of $6 \times 10^{4}$ cells per ml. We added $10 \mathrm{~mL}$ of cell suspension into each well of a 6 -well plate. Cells were cultured for $48 \mathrm{~h}$, followed by digestion with $0.25 \%$ trypsin. Cells were then harvested and dissolved in DMEM medium. After centrifugation at $1000 \mathrm{~g}$ for 5 min, cells were stained with Annexin V-FITC (Dojindo, Japan) and propidium iodide (PI), followed by flow cytometry to detect apoptotic cells.

\section{Colony formation assay}

Transfected cells were seeded into 12-well plates at a density of 1000 cells $/ \mathrm{ml}$ ( $2 \mathrm{~mL}$ per well). After 14 days culture, the cells were washed with PBS and then fixed with $3.7 \%$ methanol for 15 min and stained with $0.1 \%$ crystal violet for $30 \mathrm{~min}$ at room temperature. Clones with $>50$ cells were counted, and images of the colonies were captured by an inverted microscope (Leica, Germany).

\section{RNA Pull-down}

For the RNA pulldown assay, the Biotin RNA Labeling Mix (Roche, Basel, Switzerland) was used to transcribe and label probe-control or probe-ATB from ATB shRNA lenti vector in vitro. An RNA structure buffer (Thermo, MA, USA) was used to induce secondary structure formation from the biotin-labeled RNAs. Streptavidin beads (Thermo) were washed three times with $500 \mu \mathrm{L}$ of RNA immunoprecipitation wash buffer (Thermo) and then added to the biotinylated RNAs at $4^{\circ} \mathrm{C}$ overnight. The overnight mixture was separated by a magnetic field so that streptavidin bead-RNA complexes could be obtained. Then, lysates of cells were added to the complexes and incubated on a rotator at room temperature for $1 \mathrm{~h}$. The incubated mixture was again separated with a magnetic field so that streptavidin bead-RNA-protein complexes could be obtained. 


\section{Dual-luciferase reporter gene assay}

Luciferase vectors LINC00313 wild-type (WT) and LINC00313 mutant (MUT) were constructed by Promega. Subsequently, the cells were seeded in 24-well plates and were transfected with the LINC00313 WT or LINC00313 MUT and miR-19a-3p mimic/inhibitor or a scramble using Lipofectamine 2000 (Thermo Fisher Scientific, Inc.). The luciferase activity in the transfected cells was analyzed using the dual-luciferase assay system (Promega Corporation), following the manufacturer's protocol.

Luciferase vectors PTEN-WT and PTEN-MUT were constructed by Promega. Subsequently, the cells were seeded in 24-well plates and were transfected with the PTEN WT or PTEN MUT and miR-19a-3p mimic/inhibitor or a scramble using Lipofectamine 2000 (Thermo Fisher Scientific, Inc.). The luciferase activity in the transfected cells was analyzed using the dual-luciferase assay system (Promega Corporation), following the manufacturer's protocol.

In addition, the cells were seeded in 24-well plates and were transfected with the PTEN WT or PTEN MUT and vectors expressing LINC00313/or vector using Lipofectamine 2000 (Thermo Fisher Scientific, Inc.). The luciferase activity in the transfected cells was analyzed using the dual-luciferase assay system (Promega Corporation), following the manufacturer's protocol.

\section{Transwell Assay}

For cell invasion analysis, transwell assay was performed in this study. The upper chamber is pre-treated with $100 \mu \mathrm{L}$ of Matrigel. The cells were seeded into the upper chamber in media with $1 \% \mathrm{FBS}$, and the density was adjusted to about $1 \times 10^{6}$ cells per chamber. RPMI 1640 medium with $10 \%$ FBS was added to the lower chamber. After $24 \mathrm{~h}$ of incubation at $37^{\circ} \mathrm{C}$, the transwell chamber was rinsed twice with PBS (5 min per time), fixed by $5 \%$ glutaraldehyde at $4^{\circ} \mathrm{C}$ and stained with $0.1 \%$ crystal violet for $30 \mathrm{~min}$. The transwell chamber was washed twice with PBS and then observed under a microscope. The number of cells invading the Matrigel was regarded to be a reflection of the invasion ability.

\section{Wound healing assay}

Cells were seeded in a 6-well plate with $5.0 \times 10^{5}$ cells/well. An artificial wound was created in the confluent cell monolayer using a $200 \mu \mathrm{L}$ pipette tip. Wound closure images were taken at 0 and $24 \mathrm{~h}$ using an inverted microscope, respectively. Relative distance of wound healing was calculated.

\section{Tumor Xenograft Model}

BALB/c nude mice were purchased from Beijing Vital River Laboratory Animal Technology Company Limited (Beijing China) and housed in a specific pathogen-free facility. All animal protocols were performed in accordance with the guidelines of the Institutional Animal Care and Use Committee of Southern Medical University. For subcutaneous injections, $1 \times 10^{7}$ vectors expressing LINC00313 transfected PC3-DR cells or siRNA targeting LINC00313 transfected DU145-DR cells were resuspended with $100 \mu \mathrm{L}$ of $1 \times$ PBS and injected into right flank of BALB/nude mice (male, 4 weeks of age). Tumor 
volume and body weight $(\mathrm{g})$ was measured every 3 days. About 4 weeks later, all the animals were sacrificed, and tumor tissues were harvested, photographed, measured. Tumor volume was calculated according to the following formula: Volume $\left(\mathrm{mm}^{3}\right)=\left[\right.$ width ${ }^{2}\left(\mathrm{~mm}^{2}\right) \times$ length $\left.(\mathrm{mm})\right] / 2$. Harvested tumor tissues were placed in liquid nitrogen and then frozen at $-80^{\circ} \mathrm{C}$ or fixed in $10 \%$ buffered formalin, embedded in paraffin, sectioned and stained.

\section{Immunohistochemistry (IHC)}

Formalin-fixed, paraffin-embedded tissues were used to detect the expression of Ki67, cleaved caspase-3 and E-cadherin. The sections were incubated with anti-Ki67, anti-cleaved caspase-3 and anti-E-cadherin rabbit polyclonal antibodies (Abcam) at 1:200 dilution. Staining intensity was classified as 0 (negative), 1 (weak), 2 (moderate), and 3 (strong). Staining extent depended on the percentage of positive cells and was divided into $0(<5 \%), 1(5-25 \%), 2(26-50 \%), 3(51-75 \%)$, and $4(>75 \%)$. According to the staining intensity and the staining extent scores, the IHC result was classified as $0-1$, negative $(-) ; 2-4$, weakly positive $(+) ; 5-8$, moderately positive $(++)$; and $9-12$, strongly positive $(+++)$.

\section{Statistical analysis}

Statistical analyses were performed using SPSS 19.0 (IBM, Armonk, NY, USA). Correlations between LINC00313 expression and the clinicopathological variables were analyzed using the Pearson $\chi^{2}$ analysis. The average value of LINC00313 $>1.37$ as high expression. Survival was analyzed using the Kaplan-Meier method, and differences were evaluated using the log-rank test. The Cox proportional hazards model was used for univariate analysis or multivariate analysis to examine the potential prognostic value of different variables on overall survival (OS). Data were evaluated using ANOVA with LSD test for multiple comparisons and Student's $t$ test between two groups. $P<0.05$ was considered to indicate a statistically significant difference.

\section{Results}

\section{Identification of DE_IncRNAs and WGCNA co-expression network}

A total of 12289 IncRNAs in GSE115414 data set were obtained after reannotation. Based on the cut-off criteria, 1691 IncRNAs (including 643 upregulated and 1048 downregulated) were significantly differentially expressed, as shown in Figure $1 \mathrm{a}$ and $1 \mathrm{~b}$, and were used to construct a WGCNA coexpression network. In order to evaluate the outliers of 24 samples (8 control and 26 NAFLD), sample clustering methods were used based on the distribution of the expression values of the samples (Figure 1c), with no significant difference detected in the samples included in the WGCNA. The scale-free topology index and mean connectivity were applied during the current study to determine the soft threshold in WGCNA. The higher the scale-free topology index value was indicative of a strong probability of a non-scale feature. Power $=10$ was selected in the event of the correlation coefficient between log $(k)$ and $\log P(k)$ reached 0.9 for the first time (Figure 1d-f). Within the gene co-expression network module recognition, the maximum number of genes processed by computer was maxBlockSize $=5,000$, the 
minimum number of genes of each module was minModuleSize $=30$, and the module merge threshold was set as mergeCutHeight $=0.25$. The 31 identified modules are depicted in Figure $1 \mathrm{~g}$, whereby the different modules were marked using different colors. As can be seen from a multidimensional scaling (MDS) for gene expression data of the three modules (Figure 1h), IncRNAs in black, red, green, and turquoise modules showed dissimilar expression. The co-expression of the IncRNAs of the modules in lightgreen was relatively high. The correlation between the module IncRNAs is illustrated in Figure $1 \mathrm{i}$. In addition, three module values were calculated in each module and a clustering tree is presented in Figure $1 \mathrm{j}$. In order to obtain the IncRNA modules closely related to PCa, the relevant clinical information of the sample was extracted from the microarray, and the correlation between the above three different color modules and the 3 clinical characteristics were analyzed. The heatmap of the module and the clinical correlation is demonstrated in Figure $1 \mathrm{k}$. We observed that the grey module shared the closest correlation to PCa, which was identified to be a positive association. The correlation coefficient reached 0.9 , which was considered to be an indication that the IncRNAs in this module were highly likely to be positively affected by the development of PCa. The IncRNAs in 31 modules were used to construct a co-expression network with 609 edges and 7274 nodes (Figure 1I) using Cytoscape software and the top 10 hub genes of the network was identified using cytohubba plug-in based on 'Degree' method (Figure $1 \mathrm{~m}$ ). The hub IncRNAs above are named LINC00313, LINC00393, AC007422.1, LINC00351, AC023796, AL133166, JRKL-AS1, MIR381HG, LINC01568, and AP001116.

\section{Identification of DEGs and functional enrichment analysis in GSE55945 data set}

A total of 24442 genes in GSE55945 data set were obtained after reannotation. Based on the cut-off criteria, 663 genes (including 210 upregulated and 453 downregulated) were significantly differentially expressed, as shown in Figure $2 \mathrm{a}$ and $2 \mathrm{~b}$. We performed GSEA and GSVA analysis to further investigate the potential functions of these 663 DEGs in prostate malignant tumor. As shown in Figure 2c, these DEGs in prostate malignant tumor group were mainly enriched in "glyoxylate and dicarboxylate metabolism", "ribosome", "fructose and mannose metabolism", "homologous recombination", and "maturity onset diabetes of the young" pathways based on GSEA results. GSVA confirmed that these gene sets were close relationship with circadian rhythm mammal, basal transcription factors, abc transporters, adherens junction, AKT signaling pathway, and ether lipid metabolism (Figure 2d). The results of $\mathrm{GO}$ analysis were divided into biological processes (BP), cellular component (CC) and molecular function (MF). GO results indicated that the these DEGs were significantly enriched in $\mathrm{BP}, \mathrm{CC}$, and MF (Figure 2e and 2f). BP includes morphogenesis of an epithelium, muscle contraction and protein heterotetramerization. CC includes extracellular matrix, contractile fiber, and myofibril. And MF includes actin binding, extracellular matrix structural constituent, and chromatin DNA binding.

\section{Identification of DEGs and functional enrichment analysis in GSE102124 data set}

A total of 30905 genes in GSE102124 data set were obtained after reannotation. Based on the cut-off criteria, 572 genes (including 62 upregulated and 510 downregulated) were significantly differentially expressed, as shown in Figure $2 \mathrm{~g}$ and $2 \mathrm{~h}$. We performed GSEA and GSVA analysis to further investigate 
the potential functions of these 572 DEGs in PCa patients receiving treatment. As shown in Figure 2i, these DEGs in treated group were mainly enriched in "pyruvate metabolism", "primary bile acid biosynthesis", "glycolysis gluconeogenesis", "arginine and proline metabolism", and "nicotinate and nicotinamide metabolism" pathways based on GSEA results. GSVA confirmed that these gene sets were close relationship with protein export, glyoxylate and dicarboxylate metabolism, steroid biosynthesis, proteasome, peroxisome, $\mathrm{N}$ glycan biosynthesis, riboflavin metabolism, butanoate metabolism, sulfur metabolism, glycosylphosphatidylinositol gpi anchor biosynthesis, and valine leucine and isoleucine degradation (Figure 2j). GO results indicated that the these DEGs were significantly enriched in BP (carboxylic acid biosynthetic process, organic acid biosynthetic process, and Golgi vesicle transport), CC (endosome membrane, transport vesicle, and focal adhesion), and MF (exopeptidase activity, GDP binding, and myosin binding) (Figure 2k and 2l).

\section{WGCNA co-expression network of GSE55945 data set}

A total of 663 DEGs in GSE55945 data set were used to construct a WGCNA co-expression network. In order to evaluate the outliers of 21 samples (8 normal and 13 prostate malignant tumor), sample clustering methods were used based on the distribution of the expression values of the samples (Figure $3 a)$, with no significant difference detected in the samples included in the WGCNA. Power $=20$ was selected in the event of the correlation coefficient between $\log (k)$ and $\log P(k)$ reached 0.91 for the first time (Figure $3 b-d)$. The module merge threshold was set as mergeCutHeight $=0.25$. The 10 identified modules are depicted in Figure 3e, whereby the different modules were marked using different colors. As can be seen from a multidimensional scaling (MDS) for gene expression data of the 10 modules (Figure $3 \mathrm{f})$. The co-expression of the genes of the modules in grey was relatively high. The correlation between the module genes is illustrated in Figure $3 \mathrm{~g}$. In addition, three module values were calculated in each module and a clustering tree is presented in Figure $3 \mathrm{~h}$. In order to obtain the gene modules closely related to malignant tumor, the relevant clinical information of the sample was extracted from the microarray, and the correlation between the above three different color modules and the 2 clinical characteristics were analyzed. The heatmap of the module and the clinical correlation is demonstrated in Figure $3 \mathrm{i}$. We observed that the grey module shared the closest correlation to malignant tumor, which was identified to be a positive association. The correlation coefficient reached 0.69 , which was considered to be an indication that the genes in this module were highly likely to be positively affected by the development of PCa. The genes in 10 modules were used to construct a co-expression network with 467 edges and 1113 nodes (Figure 3j) using Cytoscape software and the top 10 hub genes of the network was identified using cytohubba plug-in based on 'Degree' method (Figure 3k). The hub IncRNAs above are named CDKN2A, PTEN, NCAM1, BDNF, KRT19, SPP1, CAV1, CALM1, KRT5, and BMP2.

\section{WGCNA co-expression network of GSE102124 data set}

A total of 572 DEGs in GSE102124 data set were used to construct a WGCNA co-expression network. In order to evaluate the outliers of 22 samples (3 control and 19 treated), sample clustering methods were used based on the distribution of the expression values of the samples (Figure 3l), with no significant 
difference detected in the samples included in the WGCNA. Power $=20$ was selected in the event of the correlation coefficient between $\log (k)$ and $\log P(k)$ reached 0.75 for the first time (Figure $3 m-0)$. The module merge threshold was set as mergeCutHeight $=0.25$. The 10 identified modules are depicted in Figure $3 p$, whereby the different modules were marked using different colors. As can be seen from a multidimensional scaling (MDS) for gene expression data of the 10 modules. The co-expression of the genes of the modules in grey was relatively high (Figure 3q). The correlation between the module genes is illustrated in Figure 3r. In addition, three module values were calculated in each module and a clustering tree is presented in Figure 3s. In order to obtain the gene modules closely related to treated group, the relevant clinical information of the sample was extracted from the microarray, and the correlation between the above three different color modules and the 2 clinical characteristics were analyzed. The heatmap of the module and the clinical correlation is demonstrated in Figure 3t. We observed that the grey module shared the closest correlation to treated group, which was identified to be a positive association. The correlation coefficient reached 0.9 , which was considered to be an indication that the genes in this module were highly likely to be positively affected by the development of PCa. The genes in 10 modules were used to construct a co-expression network with 327 edges and 1080 nodes (Figure $3 \mathrm{u}$ ) using Cytoscape software and the top 10 hub genes of the network was identified using cytohubba plugin based on 'Degree' method (Figure 3v). The hub IncRNAs above are named GAPDH, HSPA5, CANX, CDH1, HSP90B1, PTEN, SEC61A1, VEGFA, RPN1, and DNAJC3.

\section{Construction of IncRNA-associated ceRNA network}

LINC00313 was the top one hub gene of WGCNA co-expression network in GSE115414 data set. PTEN was the only one overlapping DEG between GSE55945 and GSE102124 data sets (Figure 3w).

miRNAs targeted by LINC00313 and PTEN were screened via using StarBase database. A ceRNA network was constructed using IncRNA-mRNA-miRNA pairs (Figure 3x). Furthermore, CytoNCA, a Cytoscape plugin, was used for centrality analysis of the PPI network to identify crucial genes. Therefore, LINC00313-miR-19a-3p-PTEN was identified as a core network (Figure 3y).

\section{Low LINC00313 expression was detected in PCa tumor tissues and dox-resistant PCa cells, and had a poor survival}

RT-PCR showed that LINC00313 expression is lower in PCa tumor tissues than that in matched adjacent tissues (Figure 4a). Low LINC00313 expression was correlated with a longer OS in patients with PC (Figure 4b). To investigate the relationship between LINC00313 expression and the clinicopathological features of the patients with PCa. Our results showed that LINC00313 expression was significantly associated with histological grade, preoperative PSA, Gleason score, angiolymphatic invasion, and biochemical recurrence, respectively $(P<0.05$; Table 1 and Figure $4 \mathrm{c})$ and was not associated with age, pathological stage, lymph node metastasis, and surgical margin status. Univariate and multivariate Cox regression analyses of overall survival (OS; Figure 4d and 4e) suggested that LINC00313 is an independent prognostic factor in $\mathrm{PCa}(P<0.05)$. We further found that LINC00313 expression is significantly decreased in dox-resistant PC cells (PC3-DR, and DU145-DR) (Figure 4g). 


\section{LINC00313 overexpression resulted in inhibited viability, proliferation, invasion, and migration, and induced apoptosis and cell cycle arrested at the G1 phase in dox-resistant PCa cells, in addition to the regulation of protein expression}

Vectors expressing LINC00313 were transfected into PC3-DR, and DU145-DR cells, followed by verification of high LINC00313 expression (Figure 5a). The proliferation of dox-resistant PC cells was significantly inhibited in response to LINC00313 overexpression, as determined by CCK-8 and colony formation assays (Figure 5b and 5c). Flow cytometry showed that overexpression of LINC00313 promotes apoptosis in dox-resistant PCa cells and induces cell cycle arrest at the G1 phase (Figure 5d and $5 \mathrm{e}$ ). As expected, the invasion and migration of dox-resistant PCa cells were significantly inhibited in response to LINC00313 overexpression (Figure $5 f$ and 5g). Next, we found that LINC00313 overexpression resulted in increased protein levels of p27, Bax, cleaved caspase-3/9, and E-cadherin, and decreased cyclinD1, Ki67, Bcl-2, N-cadherin, Vimentin, MMP-2, and PIP3 protein levels in PC3-DR, and DU145-DR cells (Figure 5h-i).

LINC00313 inhibition resulted in increased viability, proliferation, invasion, and migration, and suppressed apoptosis and cell cycle progression in dox-resistant PCa cells, in addition to the regulation protein expression

SiRNA targeting LINC00313\#1 or LINC00313\#2 were transfected into PC3-DR, and DU145-DR cells, followed by verification of low LINC00313 expression (Figure 6a-c). The proliferation of dox-resistant PCa cells was significantly increased in response to LINC00313 inhibition, as determined by CCK-8 and colony formation assays (Figure $6 \mathrm{~d}-\mathrm{g}$ ). Flow cytometry analysis showed that inhibition of LINC00313 inhibits apoptosis and cell cycle progression in dox-resistant PCa cells (Figure 6h and 6i). As expected, the invasion and migration of dox-resistant PCa cells were significantly upregulated in response to LINC00313 inhibition (Figure 6j-I). Next, we found that LINC00313 inhibition resulted in decreased protein levels of p27, Bax, cleaved caspase-3/-9, and E-cadherin and increased cyclinD1, Ki67, Bcl-2, N-cadherin, Vimentin, MMP-2, and PIP3 protein levels in PC3-DR, and DU145-DR cells (Figure 6k and 6I).

\section{LINC00313 affects the oncogenicity of dox-resistant PCa cells in vivo}

PC3-DR cells transfected with vectors expressing LINC00313 were subcutaneously injected into nude mice. Tumor weights and volumes were measured using an electronic balance and Vernier calipers (Figure 7a). LINC00313 overexpression resulted in reduced the tumor volume and weight (Figure 7b and 7c). Next, DU145-DR cells transfected with siRNA against LINC00313 were subcutaneously injected into nude mice; this was followed by similar analyses of tumor weight and volume (Figure 7d). Inhibition of LINC00313 increased the tumor volume and weight (Figure 7e and 7f). IHC staining showed that Ki67 expression was reduced and cleaved caspase- 3 and E-cadherin levels were increased in response to LINC00313 overexpression in the tumor tissues of nude mice, whereas Ki67 expression was increased and cleaved caspase-3 and E-cadherin levels were decreased in response to LINC00313 inhibition (Figure $7 \mathrm{~g})$. 


\section{miR-19a-3p, identified as a target miRNA of LINC00313, was upregulated in tumor tissues of patients with $\mathrm{PCa}$ and in dox-resistant PCa cells and PTEN was target gene of miR-19a-3p.}

The binding sites and modified sequence in the LINC00313 3' UTR are shown in Figure 8a. In a dualluciferase reporter assay, the luciferase activity in cells with LINC00313-WT transfection was significantly decreased in response to an miR-19a-3p mimic and was significantly increased in response to an miR19a-3p inhibitor. There were no alterations in the LINC00313-MUT-transfected cells (Figure 8b). Furthermore, RT-qPCR confirmed that the levels of miR-19a-3p in PC3-DR, and DU145-DR cells were significantly reduced in response to overexpression and were increased in response to the inhibition of LINC00313 (Figure 8c and 8d). In addition, in a biotin-labeled pull-down assay, miR-19a-3p was efficiently pulled down by LINC00313 (Figure 8e) but not by bio-LINC00313 MUT. A Spearman's correlation analysis proved that LINC00313 expression was negatively correlated with miR-19a-3p expression in 82 patients with PCa (Figure 8f). A miR-19a-3p mimic or inhibitor was transfected into PC3-DR, and DU145-DR cells, and the transfection efficiency was verified by RT-qPCR. RT-qPCR showed that the levels of miR-19a-3p in PC3-DR, and DU145-DR cells were significantly increased by the miR-19a-3p mimic and inhibited by the miR-19a-3p inhibitor (Figure 8g). The levels of miR-19a-3p in tumor tissues of patients with PCa and in dox-resistant PCa cells were significantly higher than those in normal adjacent tissues PCa cells (Figure $8 \mathrm{~h}-\mathrm{i}$ ). Additionally, miRbase and TargetScan databases were used to predict the stem-loop structure of miR-19a-3p and the binding site to the PTEN, respectively. The stem-loop structure of miR-19a-3p and the binding site to the PTEN was presented in Figure $8 \mathrm{j}$ and $8 \mathrm{k}$ using R software (RNAcofold and RNAfold) and the MFE value was $-9 \mathrm{kcal} / \mathrm{mol}$. Dual-luciferase reporter assay suggested that the luciferase activity of cells with PTEN-WT transfection was significantly decreased by miR-19a-3p mimic and the luciferase activity of cells with PTEN-WT transfection was significantly increased by miR-19a-3p inhibitor, while there was no alteration in PTEN-MUT transfected group (Figure $8 \mathrm{I}$ and $8 \mathrm{~m}$ ). To further confirm this result, miR-19a-3p mimic or inhibitor was transfected into PC3-DR, and DU145-DR cells, and transfection efficiency was evaluated by RT-qPCR and western blot assays. Further, RT-qPCR and western blot assays proved that the mRNA level of PTEN in PC3-DR, and DU145-DR cells was significantly reduced by miR19a-3p mimic, while increased by miR-19a-3p inhibitor (Figure 8n-q). A Spearman's correlation analysis proved that miR-19a-3p expression was negatively correlated with PTEN expression in 82 patients with PCa (Figure 8r). PTEN expression in tumor tissues of patients with PCa and in dox-resistant PCa cells were significantly lower than those in normal adjacent tissues PCa cells (Figure 8s and 8t).

\section{LINC00313 regulates proliferation, apoptosis, cell cycle, invasion, and migration by targeting miR-19a-3p to affect PTEN expression, thereby regulating the activation of the PI3K/Akt signaling pathway}

Firstly, western blot and RT-qPCR assays revealed that in PC3-DR cells with vectors expressing PTEN transfection, the protein and mRNA levels of PTEN was significantly increased, and in PC3-DR cells with siRNA targeting PTEN transfection, the protein and mRNA levels of PTEN was significantly decreased (Figure 9a). Next, CCK-8, colony formation, and flow cytometry assays demonstrated that the overexpression of PTEN counteracts the inhibition of proliferation and promotion of apoptosis by miR19a-3p inhibitor (Figure 9b-d). As expected, miR-19a-3p inhibitor-induced G1 phase cell cycle arrest and 
inhibition of PC3-DR cells invasion and migration were reversed by overexpression of PTEN (Figure 9e-g). We found that miR-19a-3p inhibitor resulted in increased protein levels of p27, Bax, cleaved caspase-3, and E-cadherin, and decreased cyclinD1, Ki67, Bcl-2, N-cadherin, and MMP-2 protein levels in PC3-DR cells (Figure 9h). These functions of miR-19a-3p inhibitor in the above protein expression were reversed by overexpression of PTEN. Further, in PC3-DR cells with miR-19a-3p inhibitor transfection, PTEN protein expression increased significantly and PIP3 protein expression decreased significantly, meanwhile, the phosphorylation levels of PI3K and AKT suppressed significantly. However, overexpression of PTEN abolished the effects of miR-19a-3p inhibitor on the expression of cyclinD1, p27, Ki67, Bax, Bcl-2, cleaved caspase-3, E-cadherin, N-cadherin, MMP2, PTEN, PIP3, phosphorylation (phosph)-PI3K, and phosph-AKT in PC3-DR cells with miR-19a-3p inhibitor transfection. In a dual-luciferase reporter assay, the luciferase activity in cells with PTEN-WT transfection was significantly increased in response to LINC00313 overexpression. There were no alterations in the PTEN-MUT-transfected cells (Figure 9i). Vectors expressing LINC00313 were transfected into PC3-DR cells, followed by verification of high PTEN expression and siRNA targeting LINC00313\#1 or LINC00313\#2 were transfected into PC3-DR cells, followed by verification of low PTEN expression (Figure 9j). Our results showed that PTEN silencing promoted the proliferation, invasion, and migration and inhibited the apoptosis of PC3-DR cells (Figure 9k-o). Analogously, rescue experiments showed that the functions of overexpression of LINC00313 in inhibiting cell proliferation, invasion, and migration, promoting cell apoptosis, and inducing cell cycle arrest at the G1 phase were reversed by PTEN silencing in PCa cell.

\section{Discussion}

Firstly, LINC00313 was a core IncRNA of WGCNA co-expression network about PCa. A ceRNA network of LINC00313-miR-19a-3p-PTEN was further determined using bioinformatics analysis. Next, we detected low LINC00313 expression in the tumor tissues of patients with PCa and found that low LINC00313 levels are associated with poor survival. These results suggest that LINC00313 is an anti-oncogene in PCa. More importantly, LINC00313 expression was significantly reduced in dox-resistant PCa cells. Nearly all patients with PCa develop drug resistance and refractory CRPC during treatment. Therefore, these findings substantially improve our understanding of the mechanisms underlying CRPC. Furthermore, LINC00313 overexpression in dox-resistant PCa cells resulted in inhibited viability, proliferation, invasion, and migration and induced apoptosis and cell cycle arrest at the G1 phase in vitro and inhibited tumor growth in vivo. LINC00313 inhibition in dox-resistant PCa cells promoted viability, proliferation, invasion, and migration, and inhibited apoptosis and cell cycle progression in vitro and promoted tumor growth in vivo. These results proved that overexpression of LINC00313 reduces dox resistance in PCa. These changes are closely related to changes in protein expression. The cell cycle plays an important role in cell growth and differentiation. Cell cycle regulation is mainly mediated by two types of regulatory factors (positive regulatory proteins and inhibitory regulatory proteins). Positive regulatory proteins mainly include the cell cycle-dependent kinases (CDKs) cyclinD1-CDK4/6 and cyclinDE/A-CDK2. Inhibitory regulatory proteins mainly include cyclin-dependent protein kinase inhibitors (CDKIs) [24]. CyclinD1 and p27 belong to the CDK and CDKI families, respectively, and can inhibit the cell cycle [25]. Our results 
showed that cyclinD1 expression was decreased and p27 expression was increased in response to the overexpression of LINC00313, whereas cyclinD1 expression was increased and p27 expression was decreased in response to the inhibition of LINC00313 in dox-resistant PCa cells. These results suggest that $\mathrm{LINC} 00313$ regulates the cell cycle at the $\mathrm{G} 1$ phase. $\mathrm{Ki} 67, \mathrm{Bax}, \mathrm{Bcl}-2$, and caspase-3/9 are marker proteins related to proliferation and apoptosis. High Ki67 expression in tumor tissues is related to the promotion of tumor progression [26]. As a critical apoptotic executor downstream of the caspase cascade, the activation of caspase-3 largely depends on the release of Cyt-c. Bcl-2 and Bax, members of the $\mathrm{BCl}-2$ family, are the most important regulators of apoptosis [27]. They can mediate the release of Cyt$\mathrm{C}$ and other molecules via the mitochondrial pathway. Bcl-2 and Bax not only act as upstream regulators of caspase-3, but can also act downstream as direct substrates of caspase-3 [28]. They not only interact with but also restrict each other's activity during apoptosis. Our results suggest that LINC00313 upregulates the expression of the pro-apoptotic proteins Bax and caspase-3/9 and inhibits the expression of the anti-apoptotic protein Bcl-2. E-cadherin, N-cadherin, Vimentin, and MMP-2 are markers of cell migration and invasion [29]. N-cadherin, Vimentin, and MMP-2 expression was significantly inhibited in response to the overexpression of LINC00313 in cells, and E-cadherin expression was significantly increased in response to LINC00313 overexpression. These results further prove that LINC00313 affects cell proliferation, apoptosis, migration, and invasion by regulating the expression of proteins related to the cell cycle, proliferation, apoptosis, migration, and invasion.

It has been reported that miR-19a-3p is expressed at low levels in the plasma of patients with heart failure [30]. miR-19a-3p inhibits autophagy-mediated fibrogenesis by targeting TGF- $\beta$ RII, a molecule that has not been reported in cancer. MiR-19a-3p is the target miRNA of LINC00313, and its high expression was detected in tumor tissues and dox-resistant PCa cells. Bioinformatic analysis further showed that PTEN is a target gene of miR-19a-3p. Cell function experiments confirmed that the miR-19a-3p inhibitor-induced decreases in viability, proliferation, invasion, and migration, increases in cell cycle arrest and apoptosis, and regulation of protein expression in dox-resistant PCa cells were abolished by an PTEN overexpression. And then, rescue experiments further confirmed that the LINC00313 overexpressioninduced decreases in viability, proliferation, invasion, and migration, increases in cell cycle arrest and apoptosis, and regulation of protein expression in dox-resistant PCa cells were abolished by PTEN silencing. Dual-luciferase reporter assay suggested that the luciferase activity of cells with LINC00313WT transfection was significantly decreased by miR-19a-3p mimic, the luciferase activity of cells with PTEN-WT transfection was significantly decreased by miR-19a-3p mimic, and the luciferase activity of cells with PTEN-WT transfection was significantly increased by LINC00313 overexpression. This suggests that LINC00313 functions as a ceRNA to promote PTEN by sponging miR-19a-3p, thereby regulating the proliferation and metastasis of dox-resistant PCa cells. Our previous results have shown that docetaxel resistance in human PCa cells is suppressed by blocking the PI3K/Akt signaling pathway. As an important intracellular signal transduction pathway, the PI3K/Akt signaling pathway regulates apoptosis, proliferation, migration, and invasion.

PTEN is a negative regulator of the PI3K/Akt signal transduction pathway [31]. When activated, phosphatidylinositol $(3,4,5)$-triphosphate (PIP3) can be dephosphorized, acidified, and hydrolyzed to 
phosphatidylinositol $(4,5)$-diphosphate (PIP2). PIP3 is a product of PI3K and mediates the activation of Akt. Therefore, the increased expression of PTEN can reduce the expression of PIP3 and lead to the inhibition of the PI3K/Akt signaling pathway, thereby promoting apoptosis and inhibiting cell proliferation and metastasis [32,33]. Our results showed that the overexpression of LINC00313 results in the upregulation of PTEN and downregulation of PIP3, while also manifesting overexpression of LINC00313 inhibited the activation of PI3K/AKT signaling pathway. However, a rescue experiment indicated that the functions of miR-19a-3p inhibitor in suppressing the phosphorylation levels of PI3K and AKT were reversed by overexpression of PTEN. These results suggest that LINC00313 inhibits the activation of the PTEN/PI3K/Akt signaling pathway by sponging miR-19a-3p.

In conclusion, LINC00313 expression is low in PCa tumor tissues and serves as an independent prognostic factor for patients with PCa. miR-19a-3p was identified as a target miRNA of LINC00313, and PTEN was a target of miR-19a-3p. The effects of LINC00662 overexpression on the proliferation and metastasis of dox-resistant PCa cells were reversed by an miR-19a-3p overexpression, thereby upregulating PTEN expression and blocking the PI3K/Akt signaling pathway. Therefore, LINC00313 might function as a potential anti-oncogene in CRPC.

\section{Abbreviations}

\begin{tabular}{ll} 
PPI network & protein-protein interaction network \\
\hline STRING & the Search Tool for the Retrieval Interacting Genes \\
\hline MCC & maximal clique centrality \\
\hline GEO database & Gene Expression Omnibus database \\
\hline PCa & Prostate cancer \\
\hline DEGs & The differentially expression genes \\
\hline OS & overall survival \\
\hline PTEN & Phosphatase and tensin homolog \\
\hline TCGA database & The Cancer Genome Atlas database \\
\hline DE_IncRNAs & The differentially expression IncRNAs \\
\hline GSEA & Gene set enrichment analysis \\
\hline GO & Gene Ontology \\
\hline ceRNA & competitive endogenous RNA \\
\hline GSVA & Gene set variation analysis \\
\hline WGCNA & Weighted gene co-expression network analysis \\
\hline CRPC & castration-resistant prostate cancer
\end{tabular}




\section{Declarations}

\section{Ethics approval and consent to participate}

Study were approved by the Ethics Committee of Zhujiang Hospital, Southern Medical University. The written informed consent documents were signed by all of the patients. All animal protocols were performed in accordance with the guidelines of the Institutional Animal Care and Use Committee of Southern Medical University.

\section{Consent for publication}

Not applicable.

\section{Availability of data and material}

The datasets generated/analyzed during the current study are available.

\section{Competing interests}

The authors declare that they have no competing interests.

\section{Funding}

This work was supported by grants from the National Natural Science Foundation of China (No. 81802516).

\section{Authors' contributions}

Binshen Chen, Kai Xu and Yiming Zhang wrote the main manuscript and analyzed the data. Binshen Chen, Kai Xu,Yiming Zhang,Peng Xu,and Chaoming Li performed the experiments. Jun Liu and Yawen Xu designed the study. All authors read and approved the final manuscript.

\section{Acknowledgements}

No

\section{Contributor Information}

Chen Binshen, Phone: +86-020-61643888, Email: cbshen@126.com.

Kai Xu, Email: xukay1211@163.com.

Yiming Zhang, Email: 359281481@qq.com.

Peng Xu, Email: yihuixp88@hotmail.com.

Chaoming Li, Email: 821860897@qq.com. 
Jun Liu, Email: Liujun1980s@126.com.

Yawen Xu, Email: yawenxu72@163.com.

\section{References}

1. Ye DW, Zhu Y. Prostate cancer and prostatic diseases Best of China, 2018. Prostate Cancer Prostatic Dis. 2019;22(1):1-2.

2. Nagaya N, Horie S. [Endocrine therapy for prostate cancer.]. Clin Calcium. 2018;28(11):1527-33.

3. Ahmadzadehfar H, Rahbar K, Baum RP, Seifert R, Kessel K, Bogemann M, et al. Prior therapies as prognostic factors of overall survival in metastatic castration-resistant prostate cancer patients treated with [(177)Lu]Lu-PSMA-617. A WARMTH multicenter study (the 617 trial). Eur J Nucl Med Mol Imaging. 2020.

4. Kamaldeep, Wanage G, Sahu SK, Maletha P, Adnan A, Suman S, et al. Examining Absorbed Doses of Indigenously Developed (177)Lu-PSMA-617 in Metastatic Castration-Resistant Prostate Cancer Patients at Baseline and During Course of Peptide Receptor Radioligand Therapy. Cancer Biother Radiopharm. 2020.

5. Lu C, Terbuch A, Dolling D, Yu J, Wang H, Chen Y, et al. Treatment with abiraterone and enzalutamide does not overcome poor outcome from metastatic castration-resistant prostate cancer in men with the germline homozygous HSD3B1 c.1245C genotype. Ann Oncol. 2020.

6. Li X, Zhang Y, Zhi X, Li Y, Qian K. Analysis of Clinical Efficacy of Nano-Albumin Paclitaxel Treatment for Advanced Cell Lung Cancer. J Nanosci Nanotechnol. 2020;20(10):6019-25.

7. Pathak S, Thekkekara R, Yadav U, Ahmed AT, Yim B, Lad TE, et al. Efficacy of Upfront Docetaxel With Androgen Deprivation Therapy for Castration-Sensitive Metastatic Prostate Cancer Among Minority Patients. Am J Ther. 2020.

8. de Wit R, de Bono J, Sternberg CN, Fizazi K, Tombal B, Wulfing C, et al. Cabazitaxel versus Abiraterone or Enzalutamide in Metastatic Prostate Cancer. N Engl J Med. 2019;381(26):2506-18.

9. Szarvas T, Csizmarik A, Nagy N, Keresztes D, Varadi M, Kuronya Z, et al. Molecular underpinnings of systemic treatment resistance in metastatic castration-resistant prostate cancer. Orv Hetil. 2020;161(20):813-20.

10. Jathar S, Kumar V, Srivastava J, Tripathi V. Technological Developments in IncRNA Biology. Adv Exp Med Biol. 2017;1008:283-323.

11. Luo Y, Wang X, Ma L, Ma Z, Li S, Fang X, et al. Bioinformatics analyses and biological function of IncRNA ZFPM2-AS1 and ZFPM2 gene in hepatocellular carcinoma. Oncol Lett. 2020;19(6):3677-86.

12. Shang A, Wang W, Gu C, Chen W, Lu W, Sun Z, et al. Long non-coding RNA CCAT1 promotes colorectal cancer progression by regulating miR-181a-5p expression. Aging (Albany NY). 2020;12.

13. Zhang S, Ding L, Gao F, Fan H. Long non-coding RNA DSCAM-AS1 upregulates USP47 expression through sponging miR-101-3p to accelerate osteosarcoma progression. Biochem Cell Biol. 2020. 
14. Zhang X, Chen M, Liu X, Zhang L, Ding X, Guo Y, et al. A novel IncRNA, Inc403, involved in bovine skeletal muscle moygenesis by mediating KRAS/Myf6. Gene. 2020:144706.

15. Wan B, Wu HY, Lv DJ, Zhou XM, Zhong LR, Lei B, et al. Downregulation of IncRNA PVT1 expression inhibits proliferation and migration by regulating p38 expression in prostate cancer. Oncol Lett. 2018;16(4):5160-6.

16. Shi S, Li D, Li Y, Feng Z, Du Y, Nie Y. LncRNA CR749391 acts as a tumor suppressor to upregulate KLF6 expression via interacting with miR-181a in gastric cancer. Exp Ther Med. 2020;19(1):569-78.

17. Chen D, Guo Y, Chen Y, Guo Q, Chen J, Li Y, et al. LncRNA growth arrest-specific transcript 5 targets miR-21 gene and regulates bladder cancer cell proliferation and apoptosis through PTEN. Cancer Med. 2020;9(8):2846-58.

18. Bai M, Lei Y, Wang M, Ma J, Yang P, Mou X, et al. Long Non-coding RNA SNHG17 Promotes Cell Proliferation and Invasion in Castration-Resistant Prostate Cancer by Targeting the miR-144/CD 51 Axis. Front Genet. 2020;11:274.

19. Ozgur E, Gezer U. Investigation of IncRNA H19 in prostate cancer cells and secreted exosomes upon androgen stimulation or androgen receptor blockage. Bratisl Lek Listy. 2020;121(5):362-5.

20. Zhao X, Hu X. Downregulated long noncoding RNA LINC00313 inhibits the epithelial-mesenchymal transition, invasion, and migration of thyroid cancer cells through inhibiting the methylation of ALX4. J Cell Physiol. 2019;234(11):20992-1004.

21. Wu WJ, Yin H, Hu JJ, Wei XZ. Long noncoding RNA LINC00313 modulates papillary thyroid cancer tumorigenesis via sponging miR-4429. Neoplasma. 2018;65(6):933-42.

22. Li M, Qiu M, Xu Y, Mao Q, Wang J, Dong G, et al. Differentially expressed protein-coding genes and long noncoding RNA in early-stage lung cancer. Tumour Biol. 2015;36(12):9969-78.

23. Xu N, Wu YP, Yin HB, Xue XY, Gou X. Molecular network-based identification of competing endogenous RNAs and mRNA signatures that predict survival in prostate cancer. J Transl Med. 2018;16(1):274.

24. Oguz C, Watson LT, Baumann WT, Tyson JJ. Predicting network modules of cell cycle regulators using relative protein abundance statistics. BMC Syst Biol. 2017;11(1):30.

25. Huang $C$, Zheng Y, Chen Y, Cheng Y, Jiang Y, Cai M, et al. miR-216a-5p promotes mesangial cell proliferation by targeting FoxO1 in diabetic nephropathy. Int J Clin Exp Pathol. 2019;12(1):344-55.

26. Ma Z, Lou S, Jiang Z. PHLDA2 regulates EMT and autophagy in colorectal cancer via the PI3K/AKT signaling pathway. Aging (Albany NY). 2020;12.

27. Chen $D$, Ning F, Zhang J, Tang Y, Teng X. NF-kappaB pathway took part in the development of apoptosis mediated by miR-15a and oxidative stress via mitochondrial pathway in ammonia-treated chicken splenic lymphocytes. Sci Total Environ. 2020;729:139017.

28. Gu YY, Chen MH, May BH, Liao XZ, Liu JH, Tao LT, et al. Matrine induces apoptosis in multiple colorectal cancer cell lines in vitro and inhibits tumour growth with minimum side effects in vivo via Bcl-2 and caspase-3. Phytomedicine. 2018;51:214-25. 
29. Jin T, Zhang Y, Zhang T. MiR-524-5p Suppresses Migration, Invasion, and EMT Progression in Breast Cancer Cells Through Targeting FSTL1. Cancer Biother Radiopharm. 2020.

30. Zou M, Wang F, Gao R, Wu J, Ou Y, Chen X, et al. Autophagy inhibition of hsa-miR-19a-3p/19b-3p by targeting TGF-beta R II during TGF-beta1-induced fibrogenesis in human cardiac fibroblasts. Sci Rep. 2016;6:24747.

31. Braglia L, Zavatti M, Vinceti M, Martelli AM, Marmiroli S. Deregulated PTEN/PI3K/AKT/mTOR signaling in prostate cancer: Still a potential druggable target? Biochim Biophys Acta Mol Cell Res. 2020:118731.

32. Matsuoka S, Ueda M. Mutual inhibition between PTEN and PIP3 generates bistability for polarity in motile cells. Nat Commun. 2018;9(1):4481.

33. Carnero A, Blanco-Aparicio C, Renner O, Link W, Leal JF. The PTEN/PI3K/AKT signalling pathway in cancer, therapeutic implications. Curr Cancer Drug Targets. 2008;8(3):187-98.

\section{Tables}


Table 1

Relationship between LINC00313 expression and clinical parameters

\begin{tabular}{|c|c|c|c|c|c|}
\hline Subgroup & Number & LINC00313 e & oression & $x^{2}$ & $P$-value \\
\hline & & $\operatorname{Low}(n=42)$ & High $(n=40)$ & & \\
\hline Age & & & & 1.209 & 0.272 \\
\hline$<=65$ & 42 & 24 & 18 & & \\
\hline$>65$ & 40 & 18 & 22 & & \\
\hline Histological grade & & & & 12.770 & 0.000 \\
\hline $\mathrm{G} 1+\mathrm{G} 2$ & 45 & 15 & 30 & & \\
\hline G3 & 37 & 27 & 10 & & \\
\hline Preoperative PSA & & & & 10.998 & 0.004 \\
\hline$<4 \mathrm{ng} / \mathrm{mL}$ & 38 & 12 & 26 & & \\
\hline $4-10 \mathrm{ng} / \mathrm{mL}$ & 27 & 18 & 9 & & \\
\hline$>10 \mathrm{ng} / \mathrm{mL}$ & 17 & 12 & 5 & & \\
\hline Pathological stage & & & & 0.245 & 0.621 \\
\hline $\mid+\|$ & 33 & 18 & 15 & & \\
\hline III + IV & 49 & 24 & 25 & & \\
\hline Gleason score & & & & 15.022 & 0.001 \\
\hline$<7$ & 36 & 10 & 26 & & \\
\hline$=7$ & 21 & 13 & 8 & & \\
\hline$>7$ & 25 & 19 & 6 & & \\
\hline Lymph node metastasis & & & & 1.757 & 0.185 \\
\hline negative & 41 & 18 & 23 & & \\
\hline positive & 41 & 24 & 17 & & \\
\hline Surgical margin status & & & & 0.802 & 0.370 \\
\hline negative & 39 & 22 & 17 & & \\
\hline positive & 43 & 20 & 23 & & \\
\hline Angiolymphatic invasion & & & & 8.531 & 0.003 \\
\hline
\end{tabular}




\begin{tabular}{|lccccc|}
\hline Subgroup & Number & LINC00313 expression & $\chi^{2}$ & $P$-value \\
\hline negative & 46 & 17 & 29 & & \\
positive & 36 & 25 & 11 & 4.940 & 0.026 \\
Biochemical recurrence & & & & & \\
negative & 43 & 17 & 26 & \\
positive & 39 & 25 & 14 & \\
$* P<0.05$ was considered as significant; PSA: prostate-specific antigen & & \\
\hline
\end{tabular}

Table 2

Gene primer sequences

\begin{tabular}{|ll|}
\hline Gene & Sequences \\
\hline miRNA-19a-3p-F & CATGCAACTGTGTCCCGGTTTA \\
\hline miRNA-19a-3p-R & GCTTCATTTAGAAGCCGCTCGCGAT \\
\hline miRNA-19b-3p-F & CCTGTGTGCAAACTGTCCATGCATT \\
\hline miRNA-19b-3p-R & TTGTGCATGGCTAATGCCTGCAACCA \\
\hline LINC00313-F & TGCCCCGAATGTGTCCCTCGGTCAATG \\
\hline LINC00313-R & AACTCTCAAGCGCTGTGCCCTGGG \\
\hline GAPDH-F & TGTGTCCATGTAATGTTGTTACCC \\
\hline GAPDH-R & TGGGTCGTCAAATGTCATCGTAGTC \\
\hline PTEN-F & TGCCTGATGTGTCCAACTGTTG \\
\hline PTEN-R & CCGTGAACGCTTAGCGCTAATGTGAAA \\
\hline U6-F & CGTGTGCATAGTAGATCGTCTCTTCA \\
\hline U6-R & TCCCTGTATCCCTAGCTAGTATAACT \\
\hline
\end{tabular}

Figures 

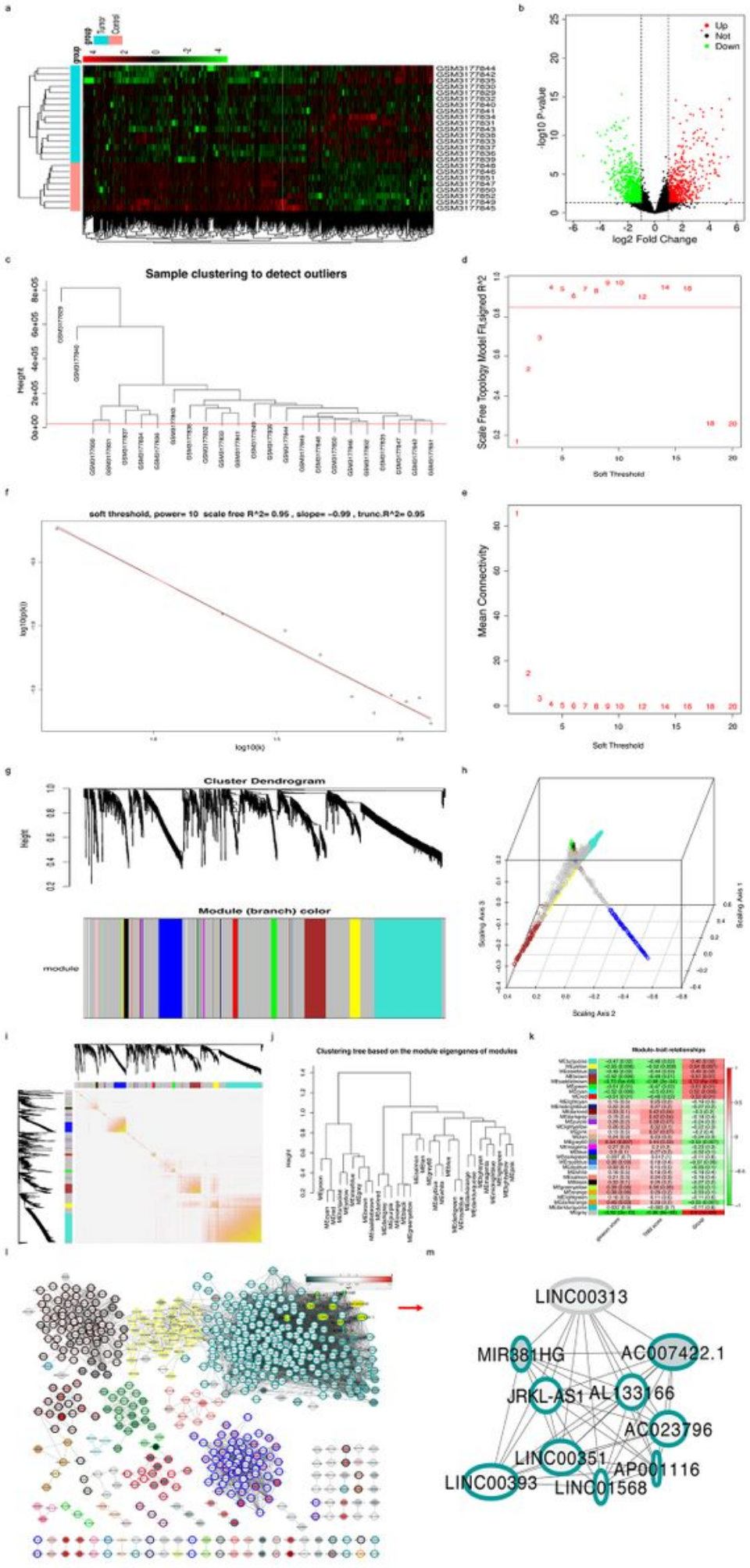

\section{Figure 1}

Identification of DE_IncRNAs and WGCNA co-expression network Heatmap (a) and volcano plot (b) were used to displayed DE_IncRNAs (643 upregulated IncRNAs and 1048 downregulated IncRNAs). Green represents upregulated DE_IncRNAs. Red represents downregulated DE_IncRNAs. Black indicates no significantly DE_IncRNAs. (c) Sample clustering dendrogram from WGCNA. (d) Analysis of the scale-free fit index for various soft-thresholding powers $(\beta)$. (e) Analysis of the mean connectivity for various soft- 
thresholding powers. In all, 10 was the most fit power value. (f) scale-free topology. (g) Cluster dendrogram of all DE-IncRNAs. (h) 3D solid figure that an MDS plot displaying expression data of genes in different modules. (i) The heatmap of co-expression gene module, the intensity of the yellow inside the heatmap represents the overlap degree of overlap, with a darker yellow representing an increased overlap. (j) Clustering tree based on the module eigengenes of modules. ( $k$ ) the correlation between WGCNA modules and the clinical features of the sample. Each row corresponds to a module, and the column corresponds to different clinical features. The numbers in the figure represent the correlation between the corresponding module and the clinical feature with the $\mathrm{P}$ value displayed in parentheses. (The red represents a positive correlation, whereas blue indicates a negative correlation). (I) PPI analysis of all DE_IncRNAs in these modules. $(\mathrm{m})$ The network of the top 10 hub IncRNAs in PPI network. 

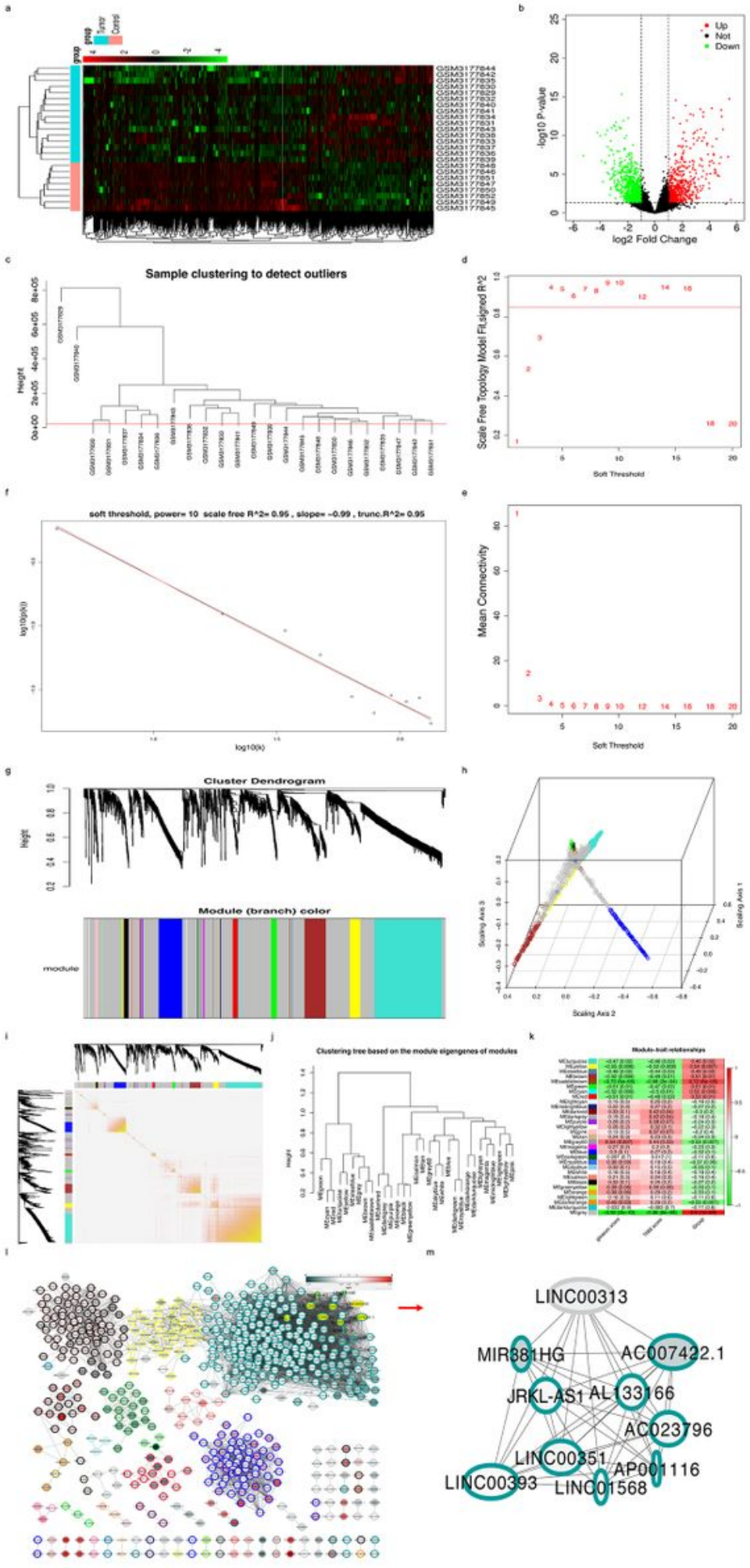

\section{Figure 1}

Identification of DE_IncRNAs and WGCNA co-expression network Heatmap (a) and volcano plot (b) were used to displayed DE_IncRNAs (643 upregulated IncRNAs and 1048 downregulated IncRNAs). Green represents upregulated DE_IncRNAs. Red represents downregulated DE_IncRNAs. Black indicates no significantly DE_IncRNAs. (c) Sample clustering dendrogram from WGCNA. (d) Analysis of the scale-free fit index for various soft-thresholding powers $(\beta)$. (e) Analysis of the mean connectivity for various soft- 
thresholding powers. In all, 10 was the most fit power value. (f) scale-free topology. (g) Cluster dendrogram of all DE-IncRNAs. (h) 3D solid figure that an MDS plot displaying expression data of genes in different modules. (i) The heatmap of co-expression gene module, the intensity of the yellow inside the heatmap represents the overlap degree of overlap, with a darker yellow representing an increased overlap. (j) Clustering tree based on the module eigengenes of modules. ( $k$ ) the correlation between WGCNA modules and the clinical features of the sample. Each row corresponds to a module, and the column corresponds to different clinical features. The numbers in the figure represent the correlation between the corresponding module and the clinical feature with the $\mathrm{P}$ value displayed in parentheses. (The red represents a positive correlation, whereas blue indicates a negative correlation). (I) PPI analysis of all DE_IncRNAs in these modules. $(\mathrm{m})$ The network of the top 10 hub IncRNAs in PPI network. 


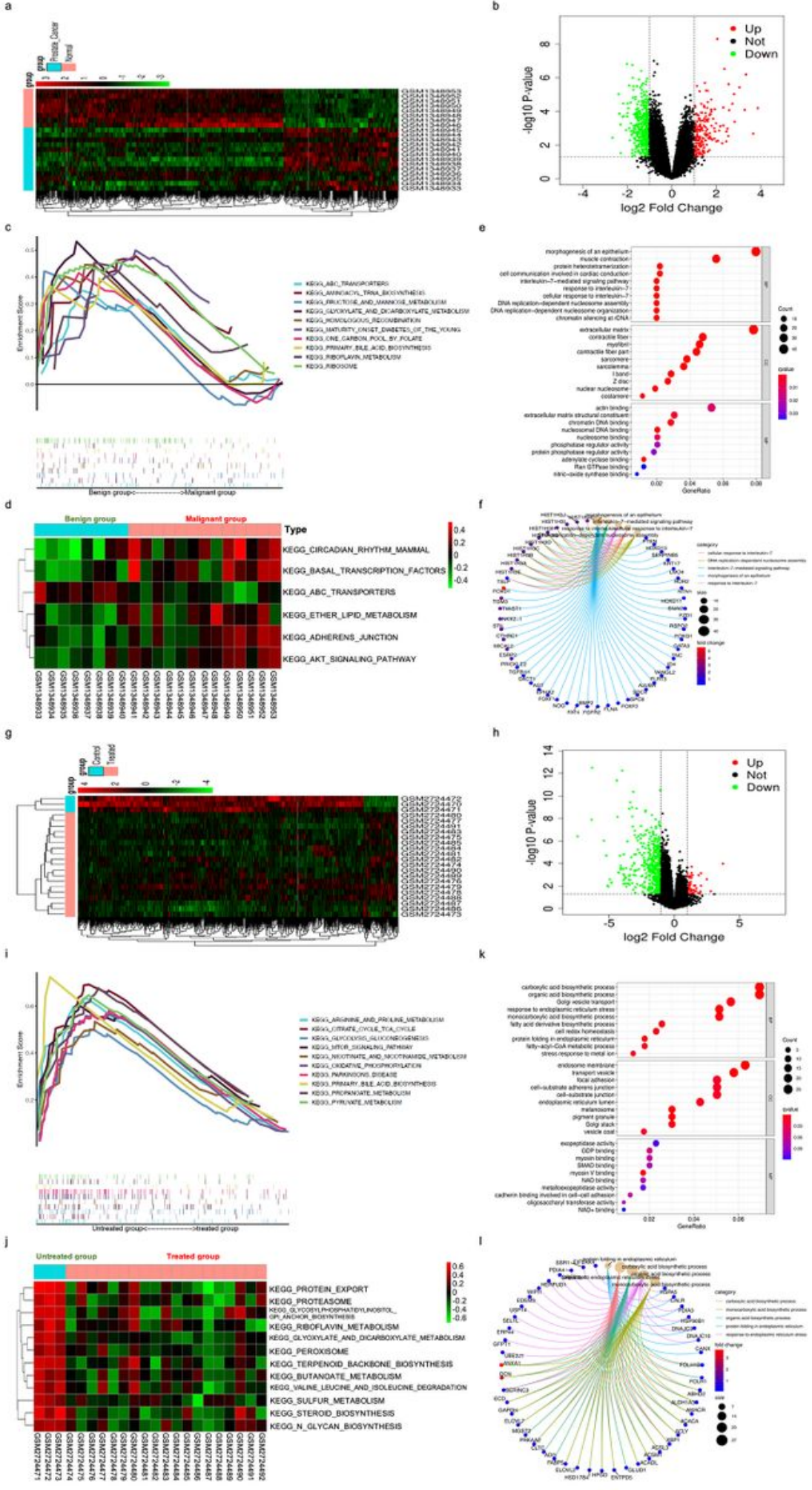

Figure 2

Identification of DEGs and functional enrichment analysis in GSE55945 and GSE102124 datasets In GSE55945 dataset, heatmap (a) and volcano plot (b) were used to displayed DEGs (210 upregulated genes and 453 downregulated genes). Green represents upregulated DEGs. Red represents downregulated DEGs. Black indicates no significantly DEGs. (c) Top 10 gene sets (according to GSEA enrichment score) enriched in prostate malignant tumor group of DEGs. (d) GSVA-derived clustering 
heatmaps of differentially expressed pathways for the DEGs. (e and f) The top $30 \mathrm{GO}$ terms of the DEGs, Biological process (BP), Cellular component (CC), and Molecular function (MF). In GSE102124 dataset, heatmap $(\mathrm{g})$ and volcano plot $(\mathrm{h})$ were used to displayed DEGs (62 upregulated genes and 510 downregulated genes). Green represents upregulated DEGs. Red represents downregulated DEGs. Black indicates no significantly DEGs. (i) Top 10 gene sets (according to GSEA enrichment score) enriched in prostate malignant tumor group of DEGs. (j) GSVA-derived clustering heatmaps of differentially expressed pathways for the DEGs. ( $k$ and $\mathrm{I}$ ) The top $30 \mathrm{GO}$ terms of the DEGs, BP, CC, and MF.

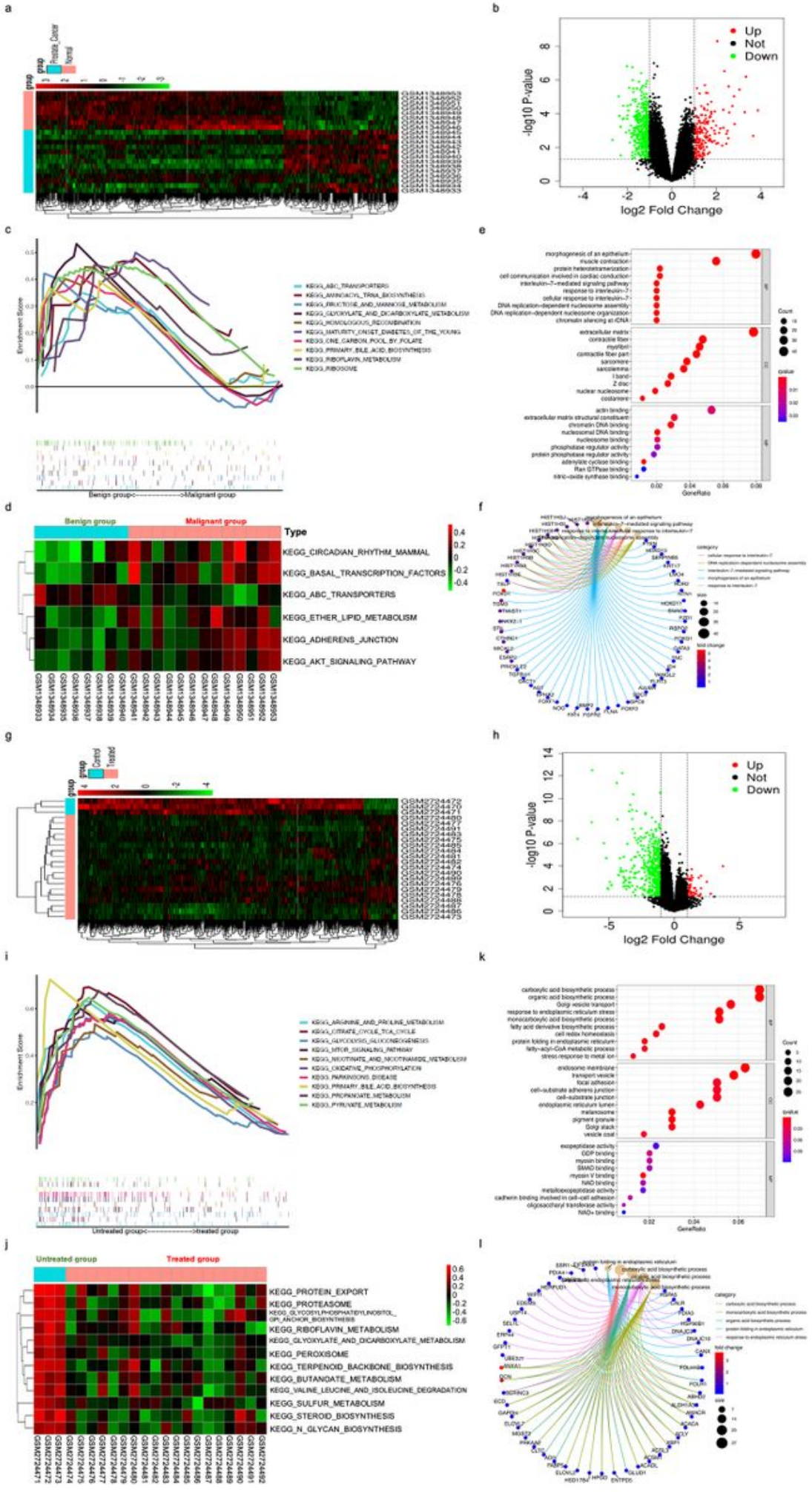




\section{Figure 2}

Identification of DEGs and functional enrichment analysis in GSE55945 and GSE102124 datasets In GSE55945 dataset, heatmap (a) and volcano plot (b) were used to displayed DEGs (210 upregulated genes and 453 downregulated genes). Green represents upregulated DEGs. Red represents downregulated DEGs. Black indicates no significantly DEGs. (c) Top 10 gene sets (according to GSEA enrichment score) enriched in prostate malignant tumor group of DEGs. (d) GSVA-derived clustering heatmaps of differentially expressed pathways for the DEGs. (e and f) The top $30 \mathrm{GO}$ terms of the DEGs, Biological process (BP), Cellular component (CC), and Molecular function (MF). In GSE102124 dataset, heatmap (g) and volcano plot $(\mathrm{h})$ were used to displayed DEGs (62 upregulated genes and 510 downregulated genes). Green represents upregulated DEGs. Red represents downregulated DEGs. Black indicates no significantly DEGs. (i) Top 10 gene sets (according to GSEA enrichment score) enriched in prostate malignant tumor group of DEGs. (j) GSVA-derived clustering heatmaps of differentially expressed pathways for the DEGs. ( $k$ and I) The top $30 \mathrm{GO}$ terms of the DEGs, BP, CC, and MF. 


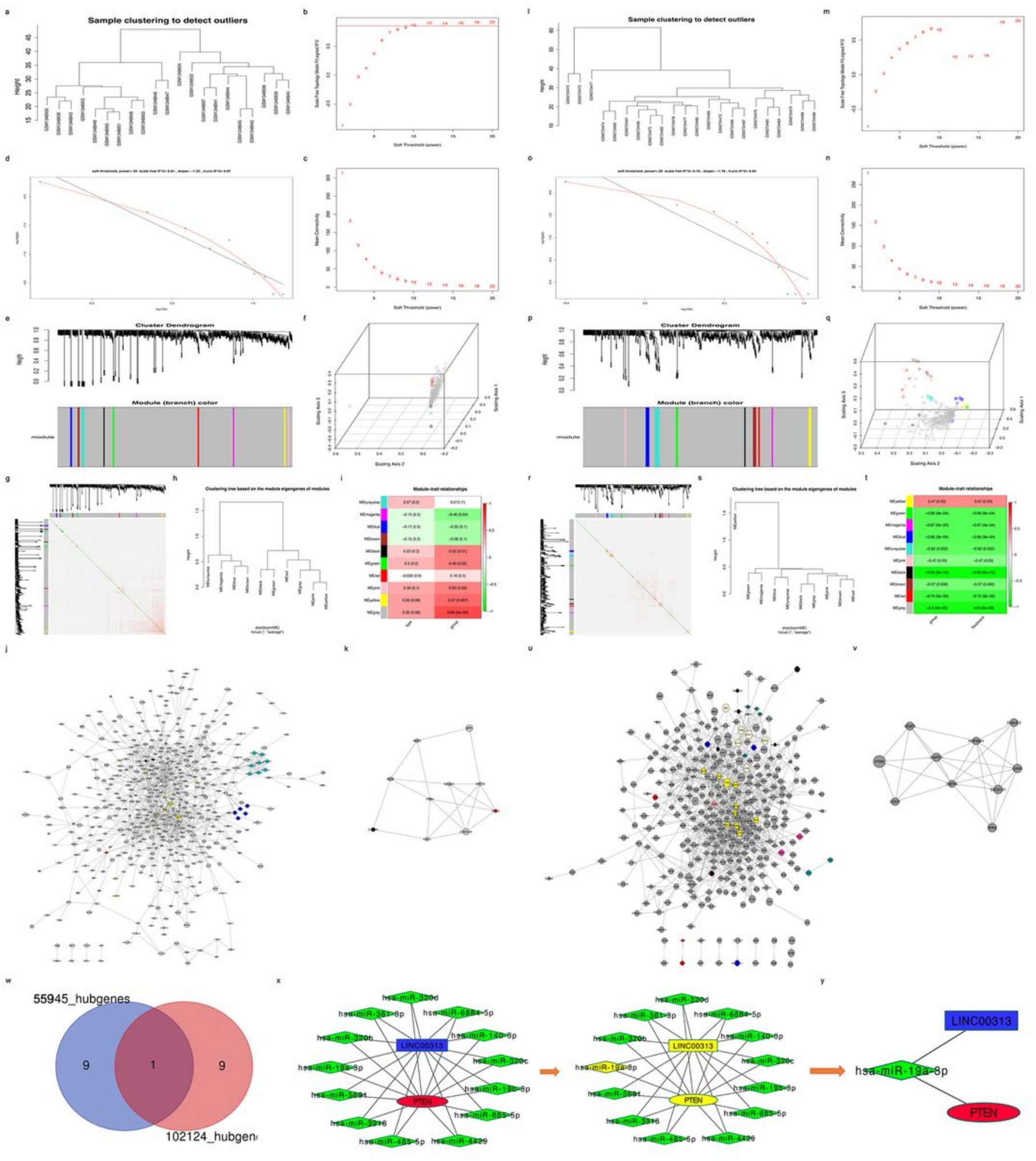

\section{Figure 3}

WGCNA co-expression network of GSE55945 and GSE102124 data sets and an ceRNA network In GSE55945 dataset, (a) Sample clustering dendrogram from WGCNA. (b) Analysis of the scale-free fit index for various soft-thresholding powers $(\beta)$. (c) Analysis of the mean connectivity for various softthresholding powers. In all, 20 was the most fit power value. (d) scale-free topology. (e) Cluster dendrogram of all DEGs. (f) 3D solid figure that an MDS plot displaying expression data of genes in 
different modules. (g) The heatmap of co-expression gene module, the intensity of the yellow inside the heatmap represents the overlap degree of overlap, with a darker yellow representing an increased overlap. (h) Clustering tree based on the module eigengenes of modules. (i) the correlation between WGCNA modules and the clinical features of the sample. Each row corresponds to a module, and the column corresponds to different clinical features. The numbers in the figure represent the correlation between the corresponding module and the clinical feature with the $\mathrm{P}$ value displayed in parentheses. (The red represents a positive correlation, whereas blue indicates a negative correlation). (j) PPI analysis of all DEGs in these modules. (k) The network of the top 10 hub genes in PPI network. In GSE102124 dataset, (I) Sample clustering dendrogram from WGCNA. (m) Analysis of the scale-free fit index for various softthresholding powers ( $\beta)$. (n) Analysis of the mean connectivity for various soft-thresholding powers. In all, 20 was the most fit power value. (o) scale-free topology. (p) Cluster dendrogram of all DEGs. (q) 3D solid figure that an MDS plot displaying expression data of genes in different modules. $(r)$ The heatmap of coexpression gene module, the intensity of the yellow inside the heatmap represents the overlap degree of overlap, with a darker yellow representing an increased overlap. (s) Clustering tree based on the module eigengenes of modules. ( $t$ ) the correlation between WGCNA modules and the clinical features of the sample. Each row corresponds to a module, and the column corresponds to different clinical features. The numbers in the figure represent the correlation between the corresponding module and the clinical feature with the $P$ value displayed in parentheses. (The red represents a positive correlation, whereas blue indicates a negative correlation). (u) PPI analysis of all DEGs in these modules. (v) The network of the top 10 hub genes in PPI network. (w) Venn plot of overlapping hub genes. (x) An ceRNA network. (y) LINC00313-miR-19a-3p-PTEN network. 


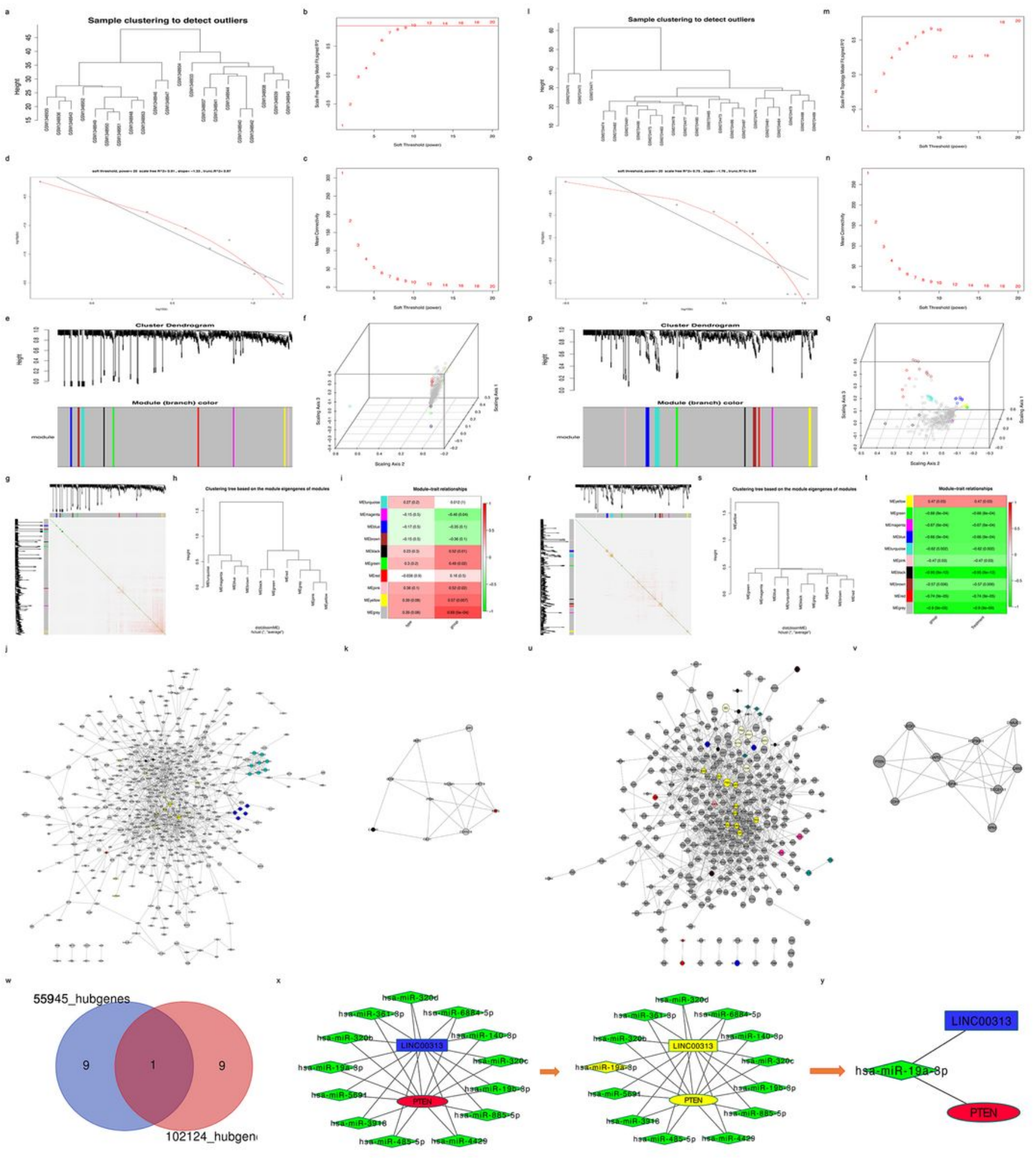

\section{Figure 3}

WGCNA co-expression network of GSE55945 and GSE102124 data sets and an ceRNA network In GSE55945 dataset, (a) Sample clustering dendrogram from WGCNA. (b) Analysis of the scale-free fit index for various soft-thresholding powers $(\beta)$. (c) Analysis of the mean connectivity for various softthresholding powers. In all, 20 was the most fit power value. (d) scale-free topology. (e) Cluster dendrogram of all DEGs. (f) 3D solid figure that an MDS plot displaying expression data of genes in 
different modules. (g) The heatmap of co-expression gene module, the intensity of the yellow inside the heatmap represents the overlap degree of overlap, with a darker yellow representing an increased overlap. (h) Clustering tree based on the module eigengenes of modules. (i) the correlation between WGCNA modules and the clinical features of the sample. Each row corresponds to a module, and the column corresponds to different clinical features. The numbers in the figure represent the correlation between the corresponding module and the clinical feature with the $\mathrm{P}$ value displayed in parentheses. (The red represents a positive correlation, whereas blue indicates a negative correlation). (j) PPI analysis of all DEGs in these modules. (k) The network of the top 10 hub genes in PPI network. In GSE102124 dataset, (I) Sample clustering dendrogram from WGCNA. (m) Analysis of the scale-free fit index for various softthresholding powers ( $\beta)$. (n) Analysis of the mean connectivity for various soft-thresholding powers. In all, 20 was the most fit power value. (o) scale-free topology. (p) Cluster dendrogram of all DEGs. (q) 3D solid figure that an MDS plot displaying expression data of genes in different modules. $(r)$ The heatmap of coexpression gene module, the intensity of the yellow inside the heatmap represents the overlap degree of overlap, with a darker yellow representing an increased overlap. (s) Clustering tree based on the module eigengenes of modules. ( $t$ ) the correlation between WGCNA modules and the clinical features of the sample. Each row corresponds to a module, and the column corresponds to different clinical features. The numbers in the figure represent the correlation between the corresponding module and the clinical feature with the $P$ value displayed in parentheses. (The red represents a positive correlation, whereas blue indicates a negative correlation). (u) PPI analysis of all DEGs in these modules. (v) The network of the top 10 hub genes in PPI network. (w) Venn plot of overlapping hub genes. (x) An ceRNA network. (y) LINC00313-miR-19a-3p-PTEN network. 
a
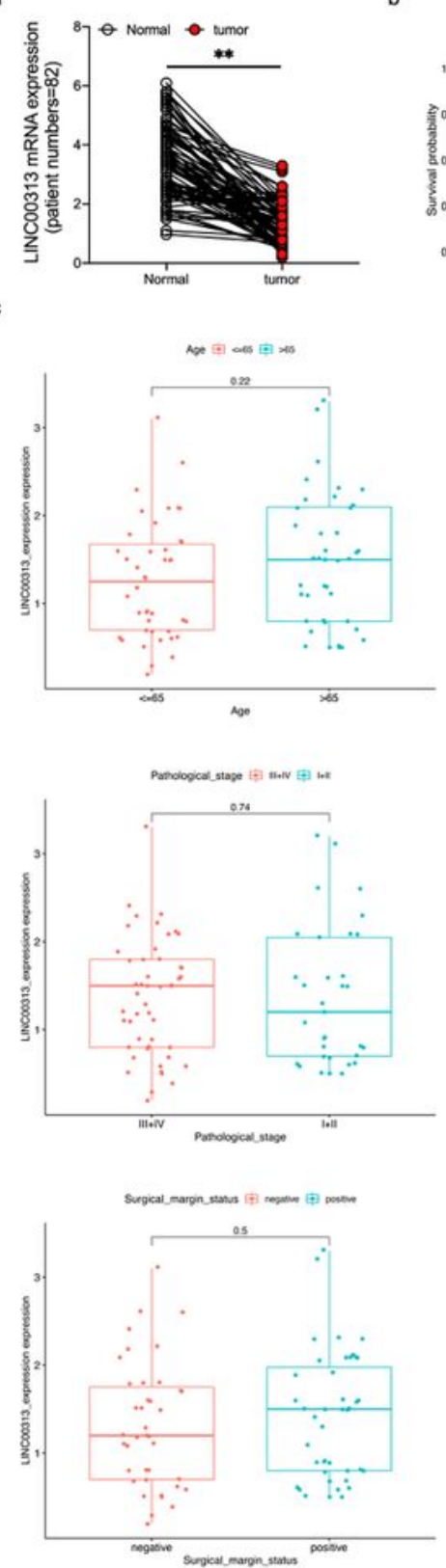

d

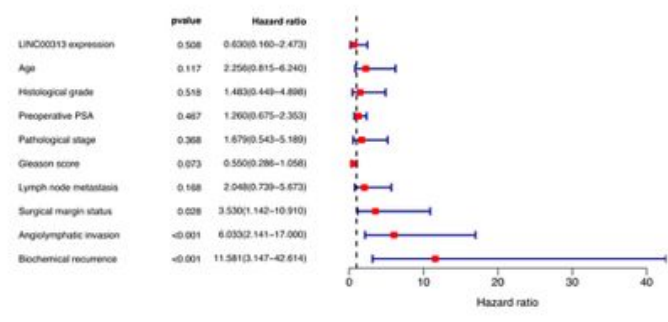

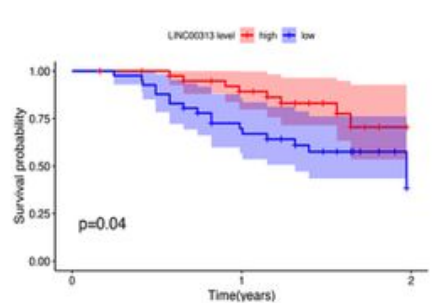
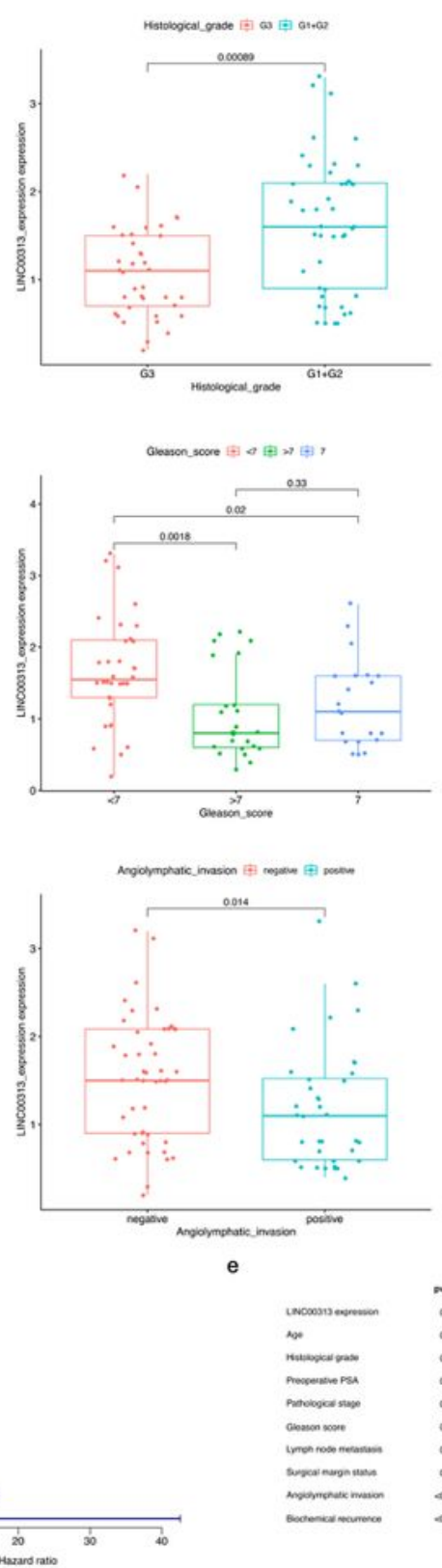
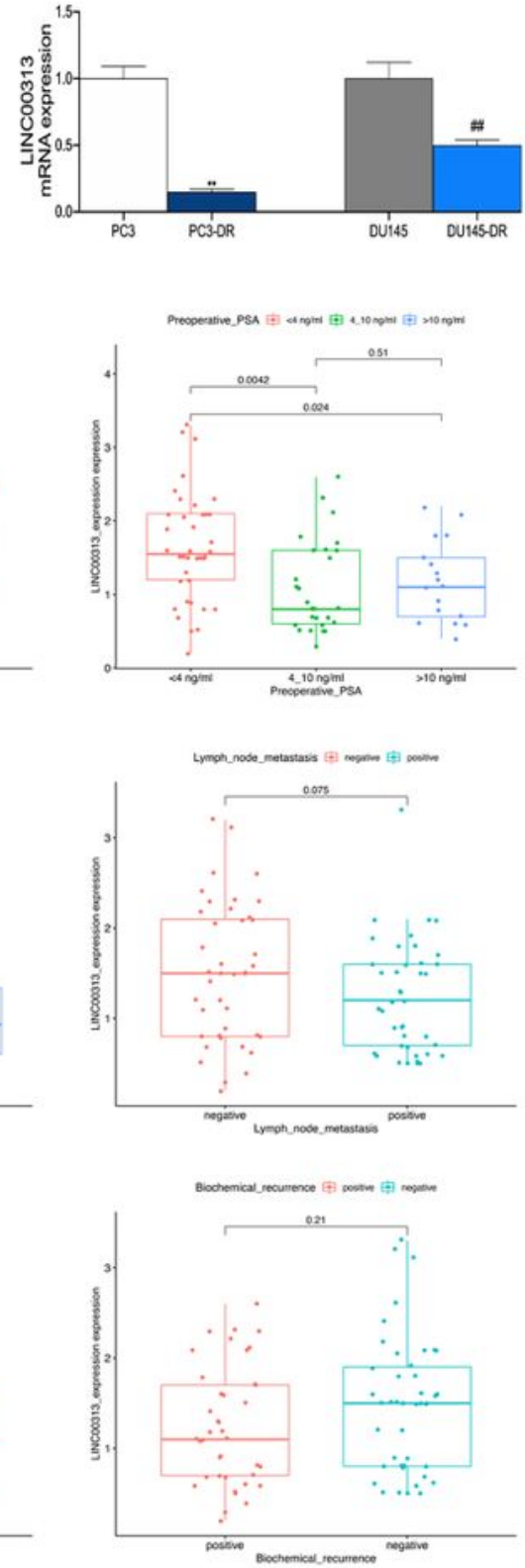

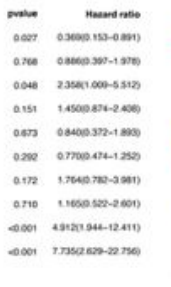

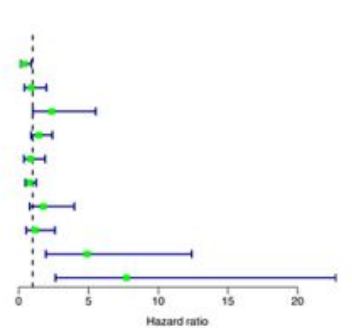

\section{Figure 4}

Low LINC00313 expression was detected in PCa tumor tissues and dox-resistant PCa cells, and had a poor survival (a) The mRNA level of LINC00313 between normal adjacent group and PC tumor group was detected by RT-qPCR assay. (b) Survival curve was performed by using the Kaplan-Meier method, and differences between the curves in LINC00313 high expression group and LINC00313 low expression group were determined by log-rank test. (c) Statistical graphs of the relationship between LINC00313 
expression and histological grade, preoperative PSA, Gleason score, angiolymphatic invasion, biochemical recurrence, age, pathological stage, lymph node metastasis, and surgical margin status. (d) Forest plots showing the univariate analysis of COX regression for overall survival. (e) Forest plots showing the multivariate analysis of COX regression for overall survival. (f) The mRNA level of LINC00313 between PC/or DU145 cells and PC-DR/or DU145-DR cells was detected by RT-qPCR assay. GAPDH was used as a load control Data are presented as the mean \pm standard deviation. ${ }^{*} \mathrm{P}<0.01$ vs. tumor group/PC3 group, \#\#P $<0.01$ vs. DU145 group.

a

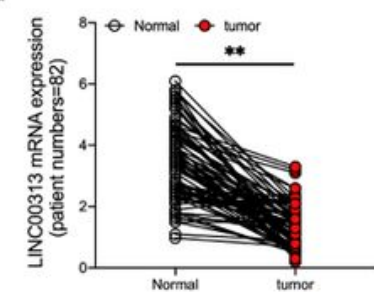

c
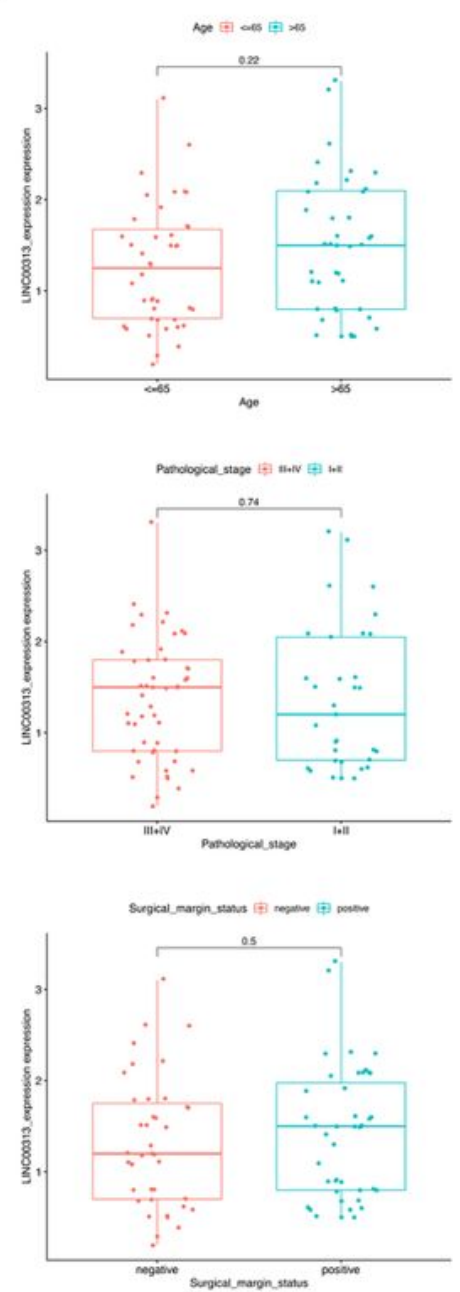

d

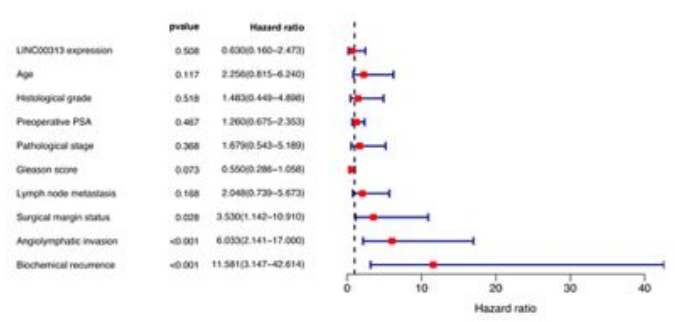

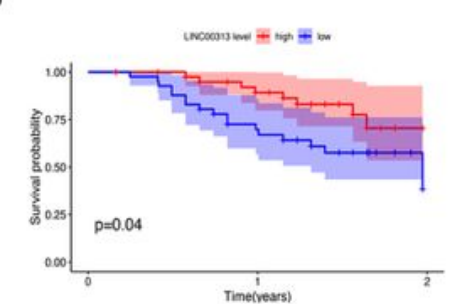
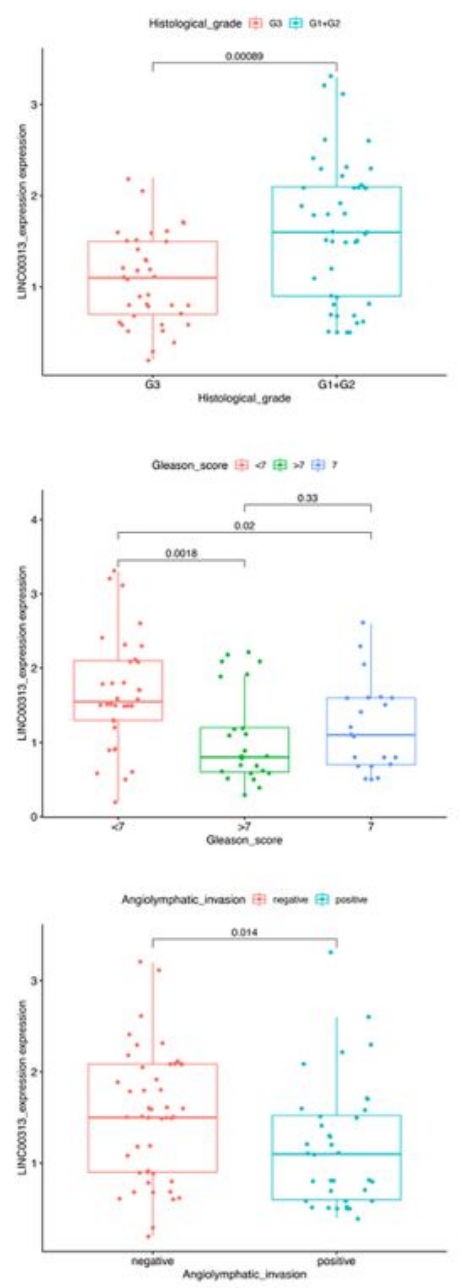

e
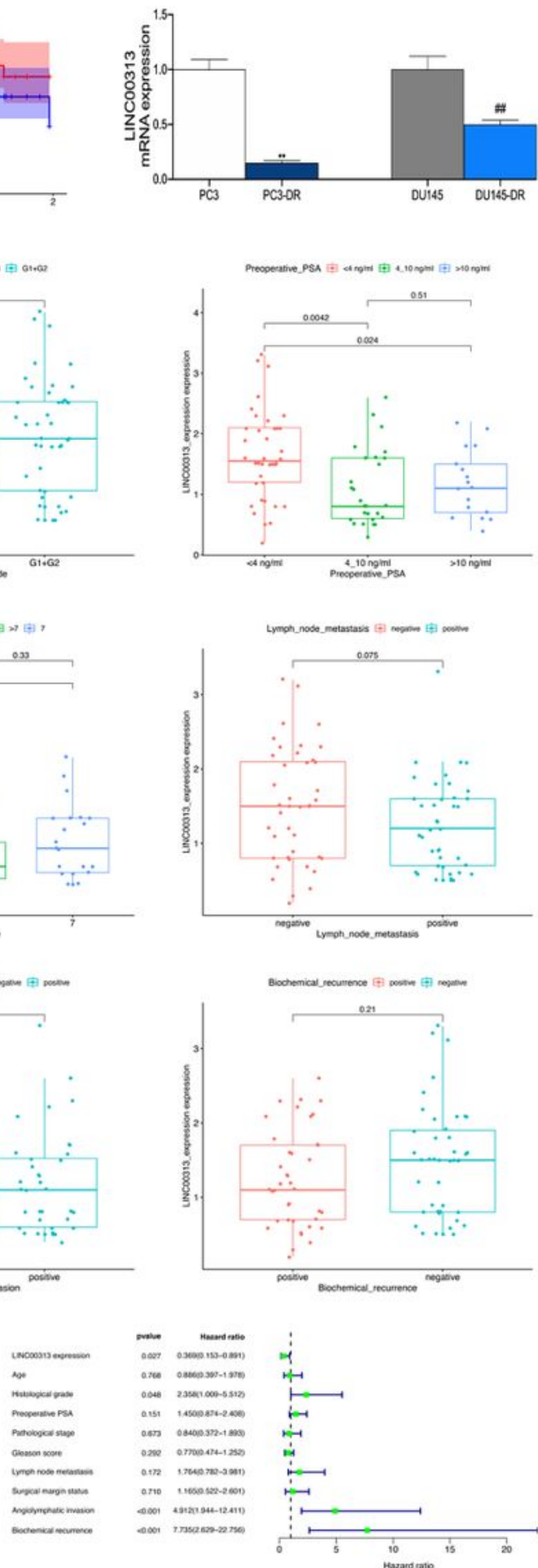

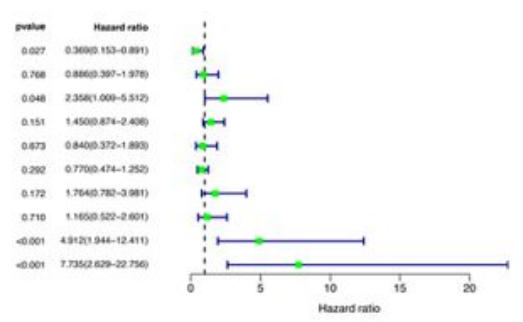




\section{Figure 4}

Low LINC00313 expression was detected in PCa tumor tissues and dox-resistant PCa cells, and had a poor survival (a) The mRNA level of LINC00313 between normal adjacent group and PC tumor group was detected by RT-qPCR assay. (b) Survival curve was performed by using the Kaplan-Meier method, and differences between the curves in LINC00313 high expression group and LINC00313 low expression group were determined by log-rank test. (c) Statistical graphs of the relationship between LINC00313 expression and histological grade, preoperative PSA, Gleason score, angiolymphatic invasion, biochemical recurrence, age, pathological stage, lymph node metastasis, and surgical margin status. (d) Forest plots showing the univariate analysis of COX regression for overall survival. (e) Forest plots showing the multivariate analysis of COX regression for overall survival. (f) The mRNA level of LINC00313 between PC/or DU145 cells and PC-DR/or DU145-DR cells was detected by RT-qPCR assay. GAPDH was used as a load control Data are presented as the mean \pm standard deviation. ${ }^{\star *} \mathrm{P}<0.01$ vs. tumor group/PC3 group, \#\#P $<0.01$ vs. DU145 group.

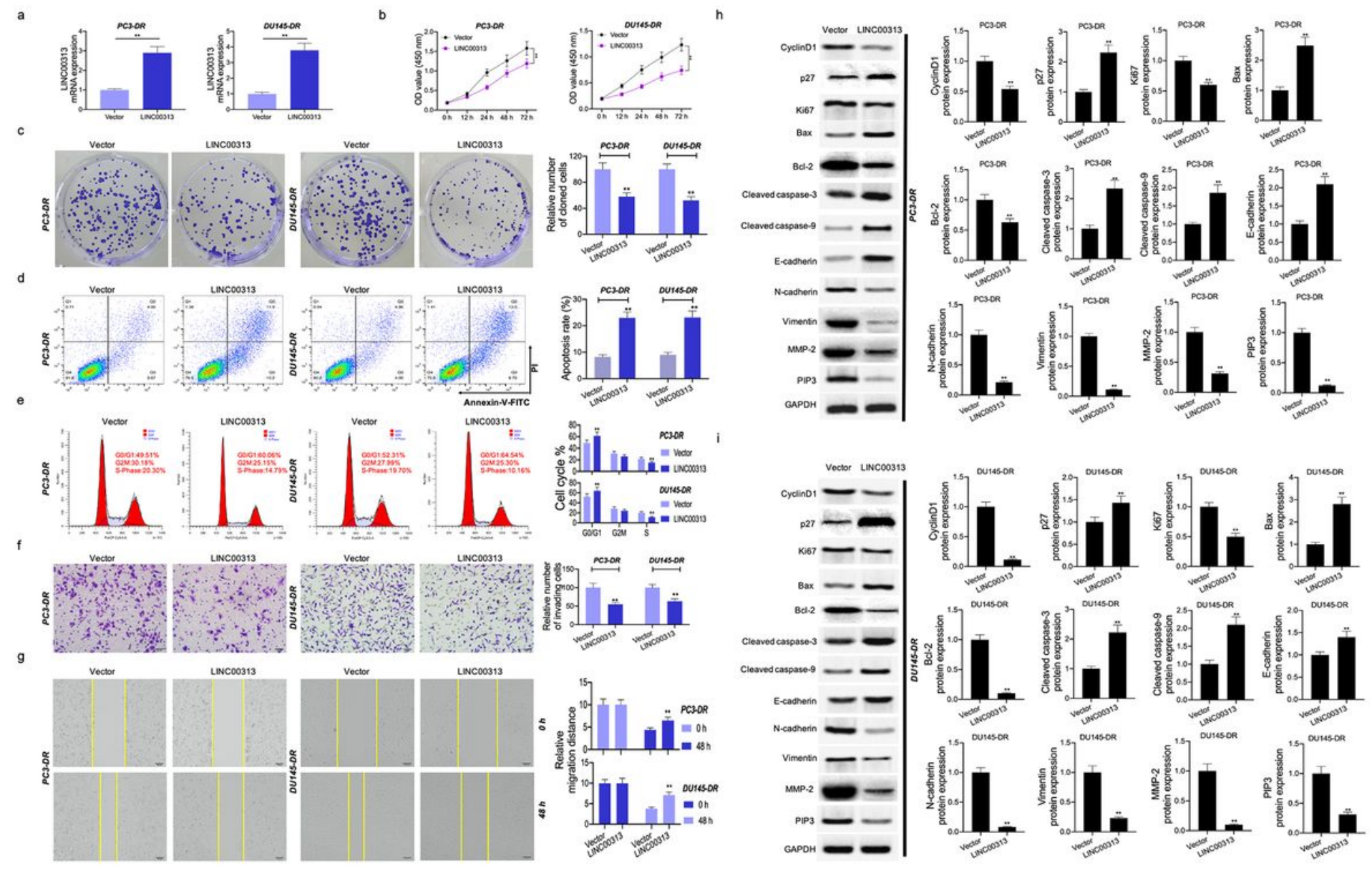

Figure 5

LINC00313 overexpression resulted in inhibited viability, proliferation, invasion, and migration, and induced apoptosis and cell cycle arrested at the G1 phase in dox-resistant PCa cells, in addition to the regulation of protein expression After vectors expressing LINC00313 was transfected into PC3-DR, and DU145-DR cells, (a) LINC00313 mRNA expression was detected by RT-qPCR assay, (b) cell viability was 
detected by CCK-8 assay, (c) cell proliferation was detected by colony formation assay, ( $d$ and e) cell apoptosis and cell cycle were detected by flow cytometry analysis, (f) cell invasion was detected by transwell assay, (g) cell migration was detected by Scratch-Wound assay, and the protein levels of cyclinD1, p27, Ki67, Bax, Bcl-2, cleaved caspase-3/-9, E-cadherin, N-cadherin, Vimentin, MMP-2, and PIP3 in (h) PC3-DR cells, and (i) DU145-DR cells were detected by detected by western blot assay. GAPDH was used as a load control Data are presented as the mean \pm standard deviation. ${ }^{*} \mathrm{P}<0.01$ vs. vector group.

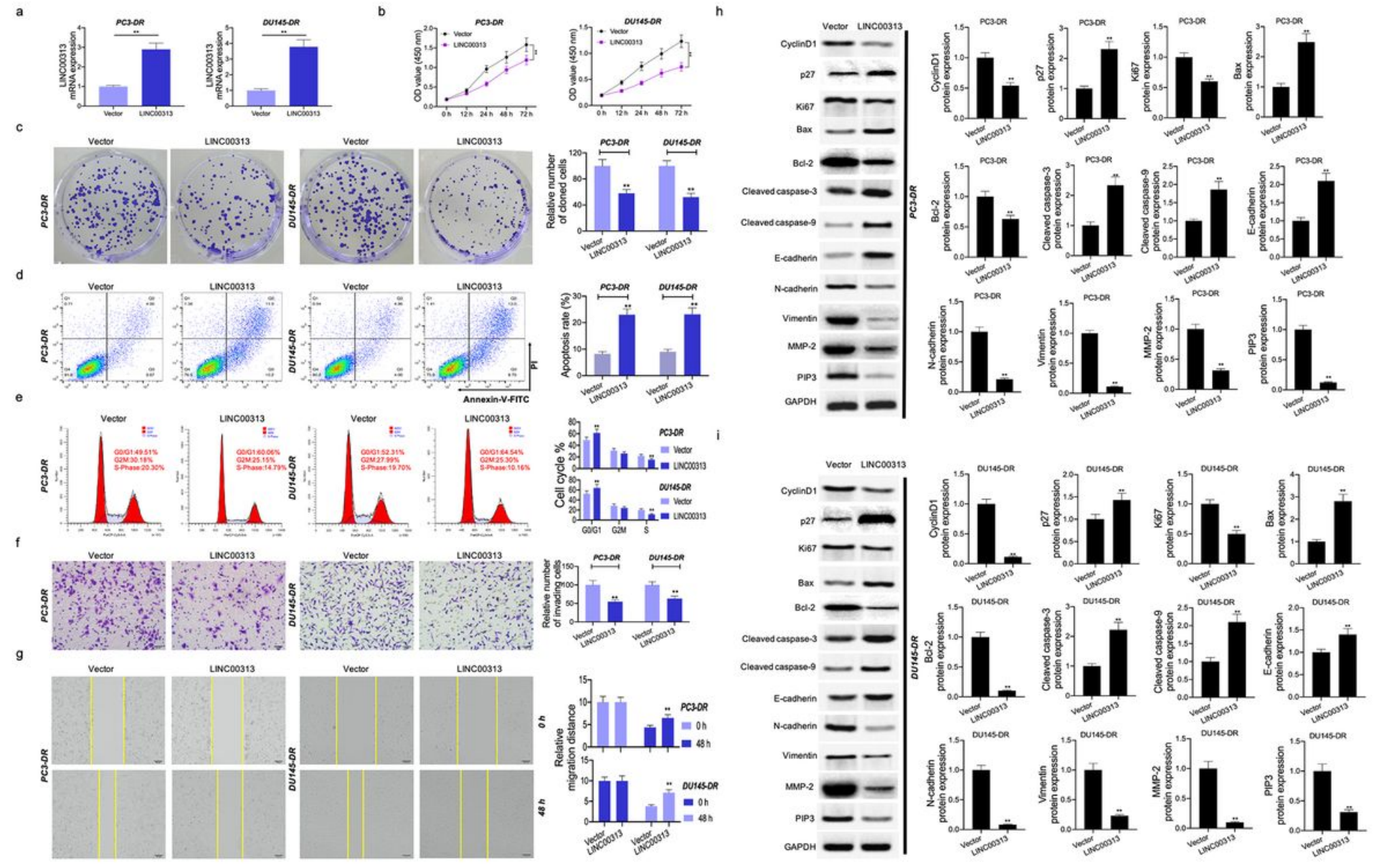

\section{Figure 5}

LINC00313 overexpression resulted in inhibited viability, proliferation, invasion, and migration, and induced apoptosis and cell cycle arrested at the G1 phase in dox-resistant PCa cells, in addition to the regulation of protein expression After vectors expressing LINC00313 was transfected into PC3-DR, and DU145-DR cells, (a) LINC00313 mRNA expression was detected by RT-qPCR assay, (b) cell viability was detected by CCK-8 assay, (c) cell proliferation was detected by colony formation assay, ( $d$ and e) cell apoptosis and cell cycle were detected by flow cytometry analysis, (f) cell invasion was detected by transwell assay, (g) cell migration was detected by Scratch-Wound assay, and the protein levels of cyclinD1, p27, Ki67, Bax, Bcl-2, cleaved caspase-3/-9, E-cadherin, N-cadherin, Vimentin, MMP-2, and PIP3 in (h) PC3-DR cells, and (i) DU145-DR cells were detected by detected by western blot assay. GAPDH was used as a load control Data are presented as the mean \pm standard deviation. ${ }^{*} \mathrm{P}<0.01$ vs. vector group. 

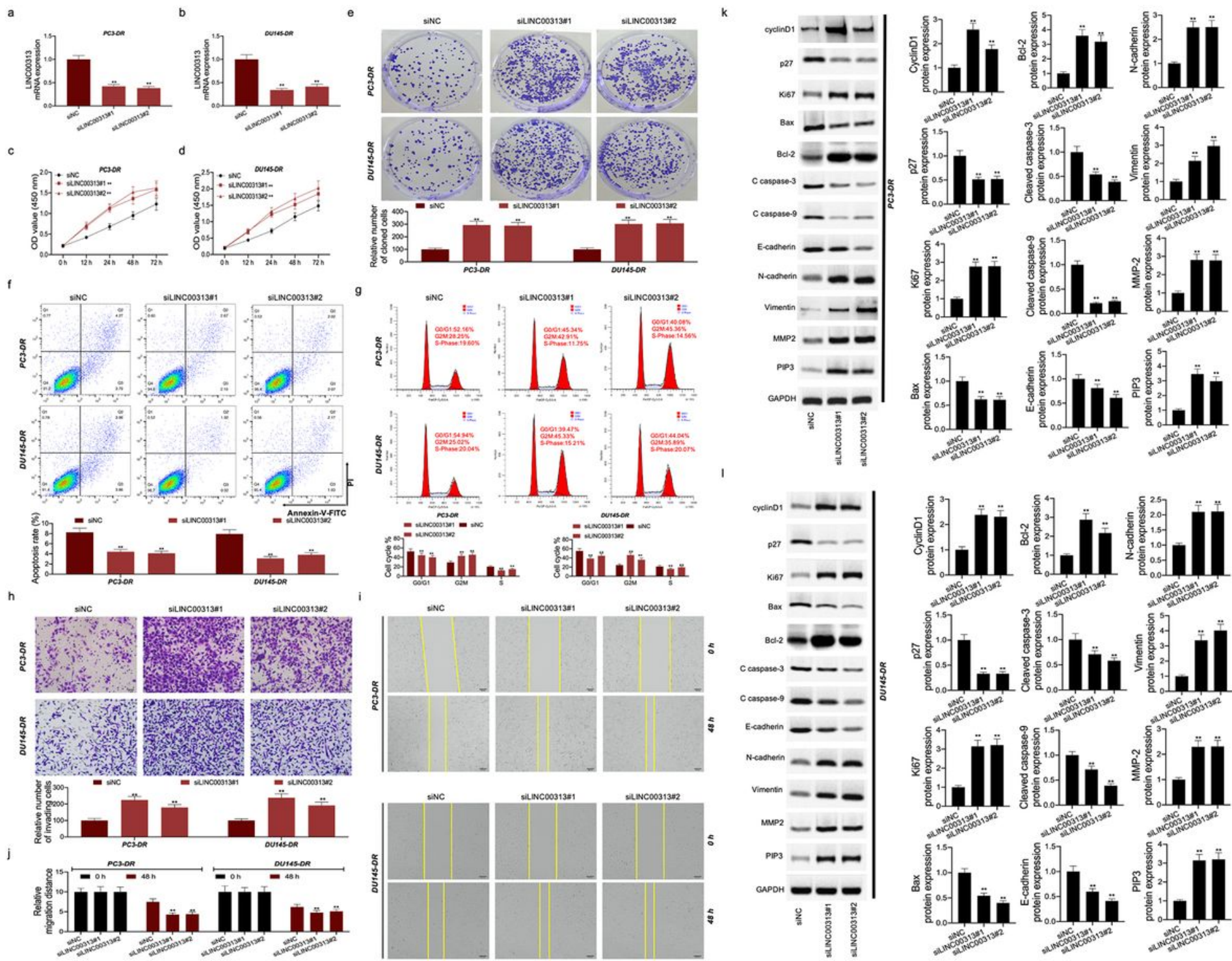
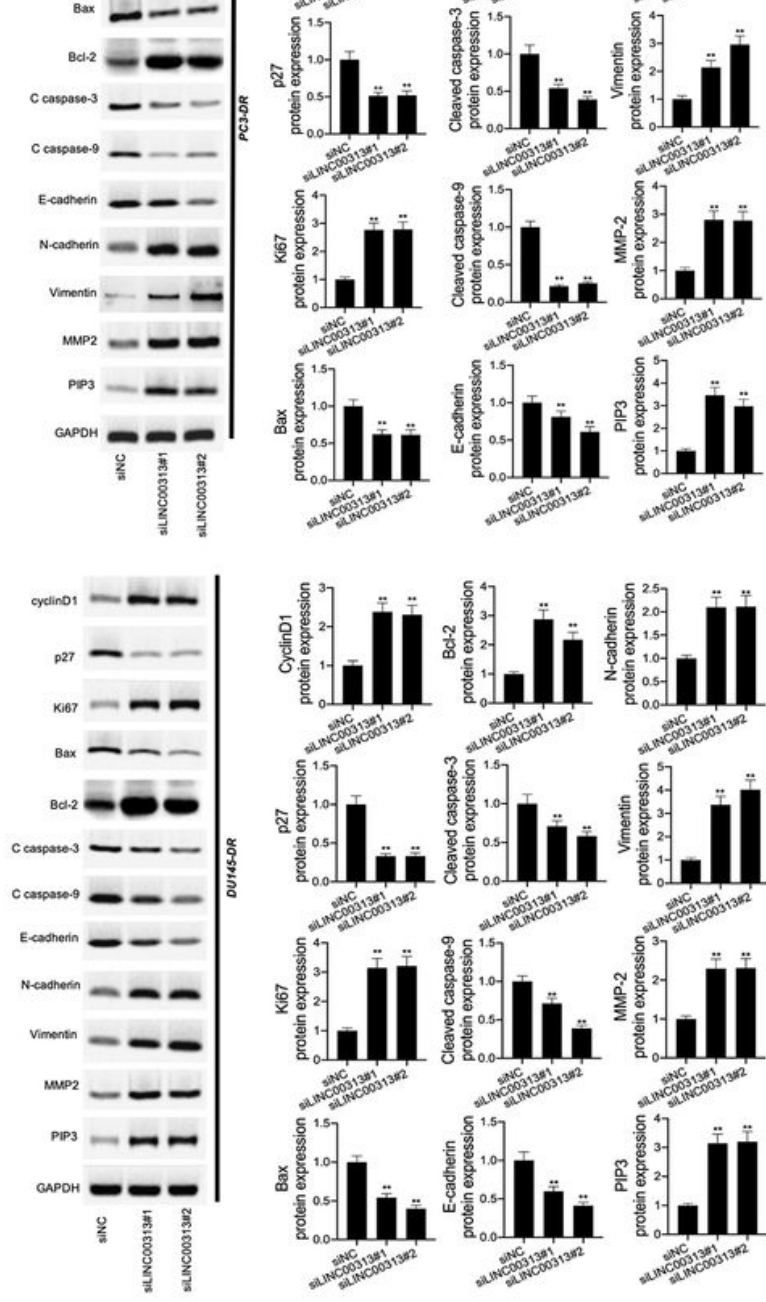

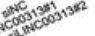

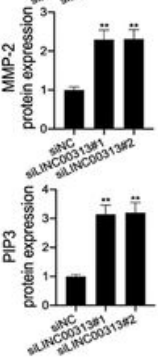

Figure 6

LINC00313 inhibition resulted in increased viability, proliferation, invasion, and migration, and suppressed apoptosis and cell cycle progression in dox-resistant PCa cells, in addition to the regulation protein expression After siRNA targeting LINC00313\#1 or LINC00313\#2 was transfected into PC3-DR, and DU145-DR cells, $(a, b)$ LINC00313 mRNA expression was detected by RT-PCR assay, $(c, d)$ cell viability was detected by CCK-8 assay, (e) cell proliferation was detected by colony formation assay, (f) cell apoptosis was detected by flow cytometry analysis, (g) cell cycle was detected by flow cytometry analysis, (h) cell invasion was detected by transwell assay, and ( $i$ and $j$ ) cell migration was detected by Scratch-Wound assay, and the protein levels of cyclinD1, p27, Ki67, Bax, Bcl-2, cleaved caspase-3/-9, Ecadherin, N-cadherin, Vimentin, MMP-2, and PIP3 in (k) PC3-DR cells, and (I) DU145-DR cells were detected by detected by western blot assay. GAPDH was used as a load control Data are presented as the mean \pm standard deviation. ${ }^{*} \mathrm{P}<0.01$ vs. siNC group. 

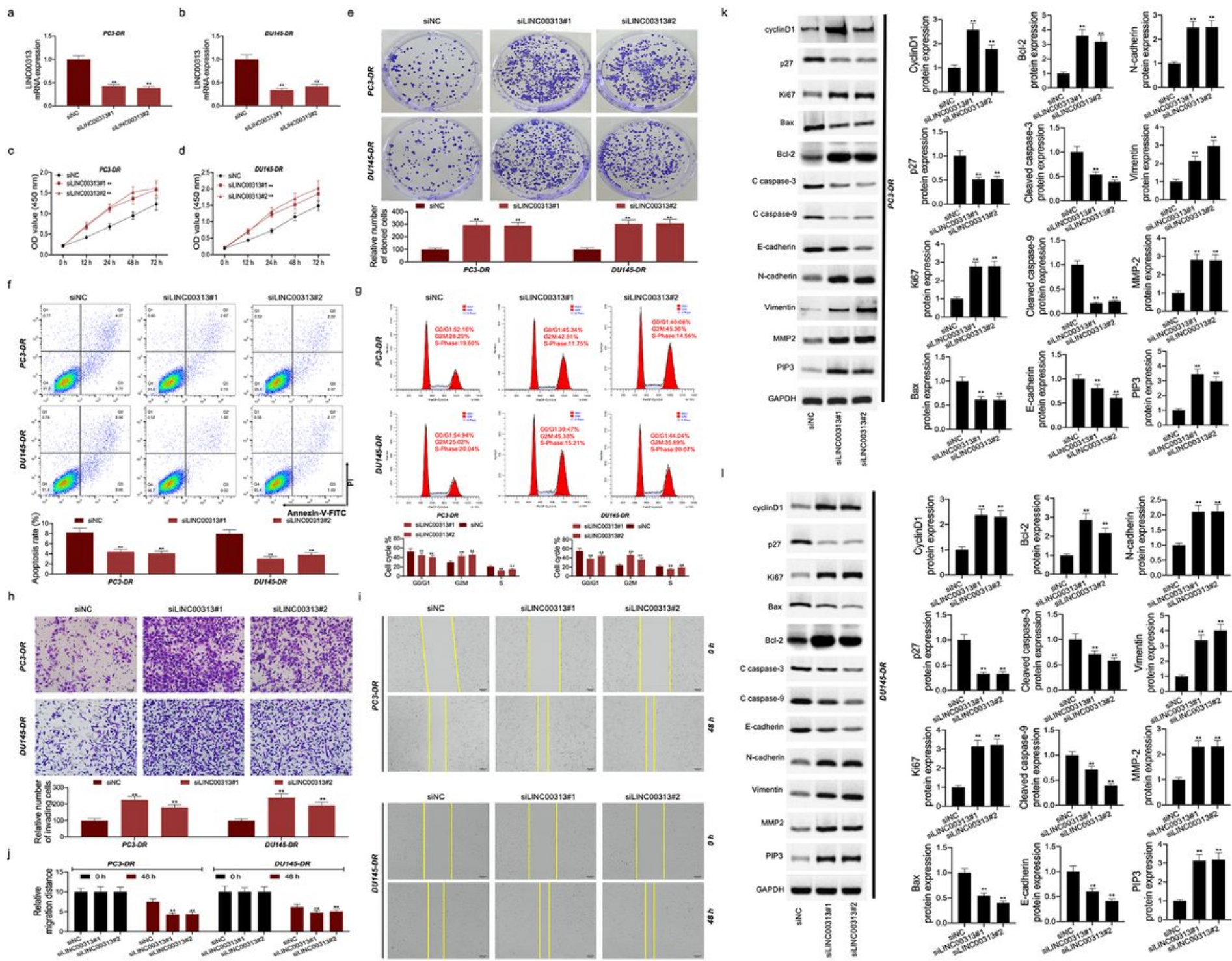
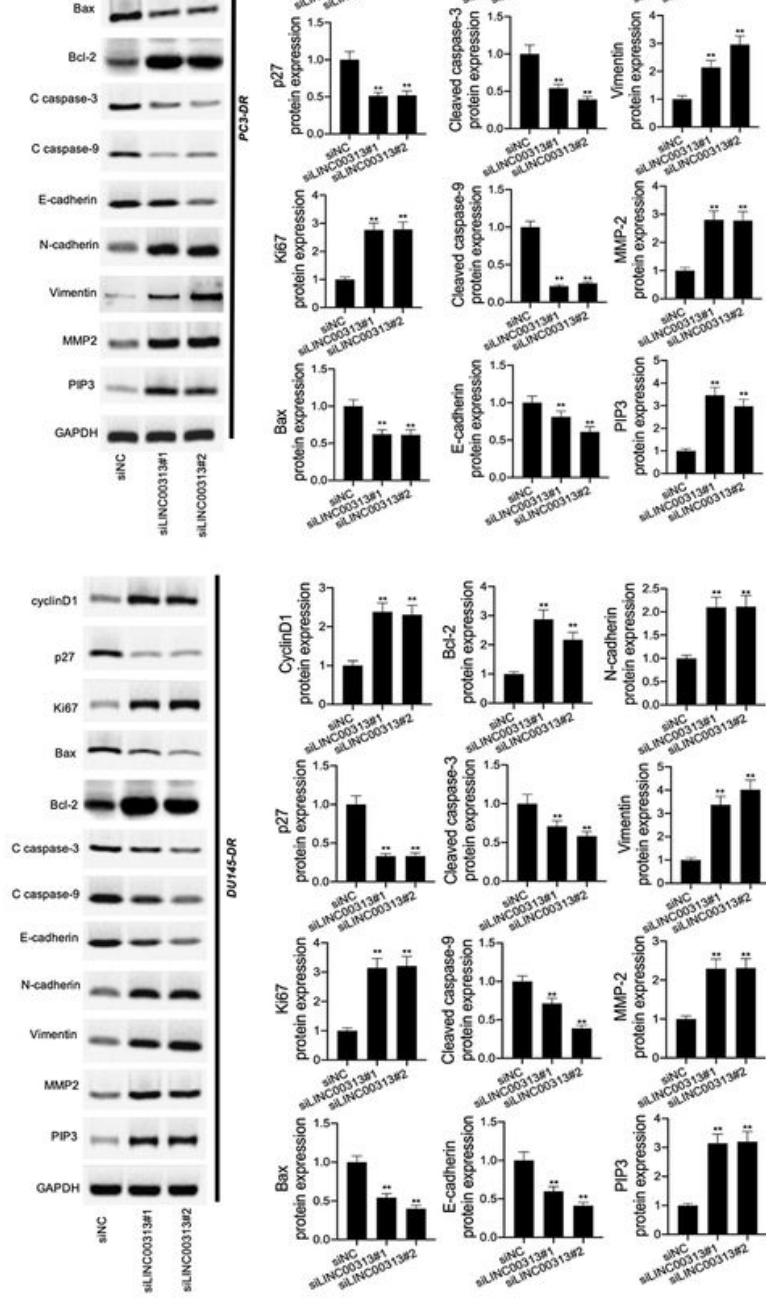

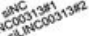

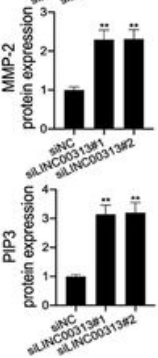

Figure 6

LINC00313 inhibition resulted in increased viability, proliferation, invasion, and migration, and suppressed apoptosis and cell cycle progression in dox-resistant PCa cells, in addition to the regulation protein expression After siRNA targeting LINC00313\#1 or LINC00313\#2 was transfected into PC3-DR, and DU145-DR cells, $(a, b)$ LINC00313 mRNA expression was detected by RT-PCR assay, $(c, d)$ cell viability was detected by CCK-8 assay, (e) cell proliferation was detected by colony formation assay, (f) cell apoptosis was detected by flow cytometry analysis, (g) cell cycle was detected by flow cytometry analysis, (h) cell invasion was detected by transwell assay, and ( $i$ and $j$ ) cell migration was detected by Scratch-Wound assay, and the protein levels of cyclinD1, p27, Ki67, Bax, Bcl-2, cleaved caspase-3/-9, Ecadherin, N-cadherin, Vimentin, MMP-2, and PIP3 in (k) PC3-DR cells, and (I) DU145-DR cells were detected by detected by western blot assay. GAPDH was used as a load control Data are presented as the mean \pm standard deviation. ${ }^{*} \mathrm{P}<0.01$ vs. siNC group. 
a

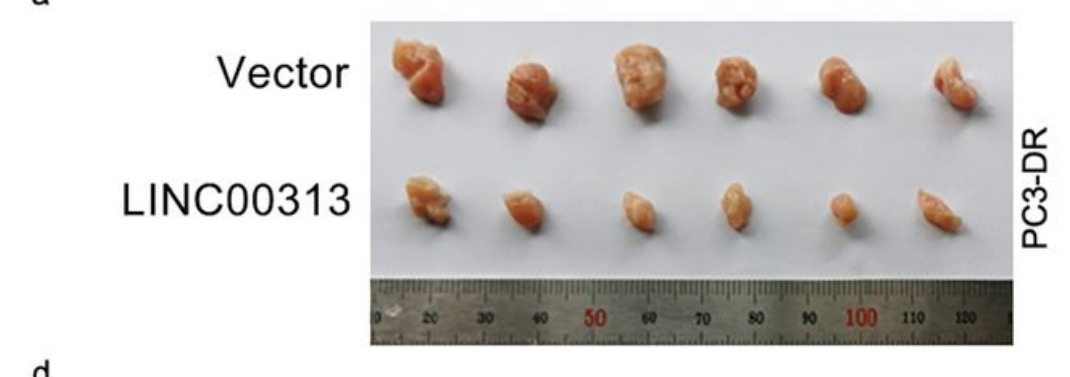

d

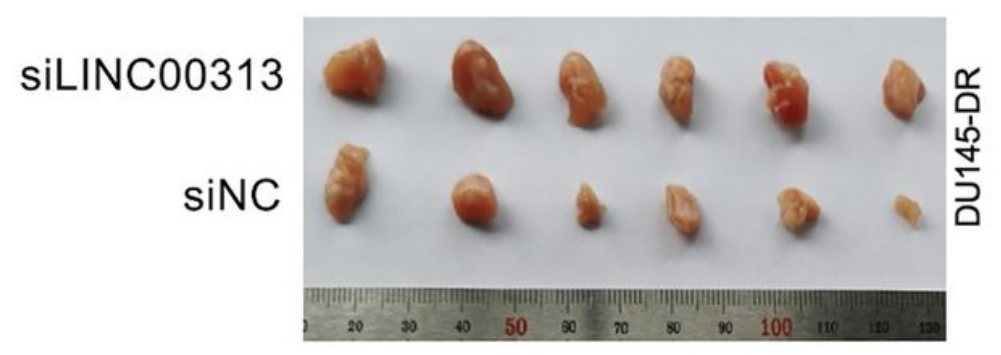

b

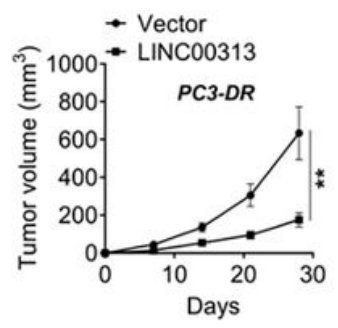

e

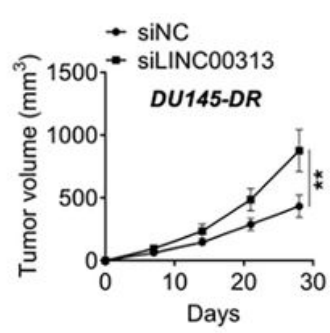

C

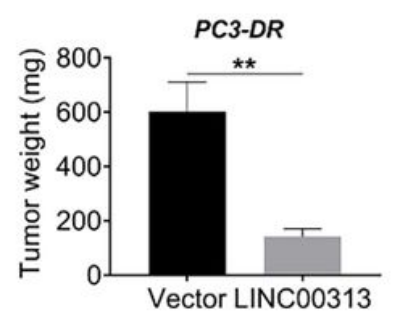

f

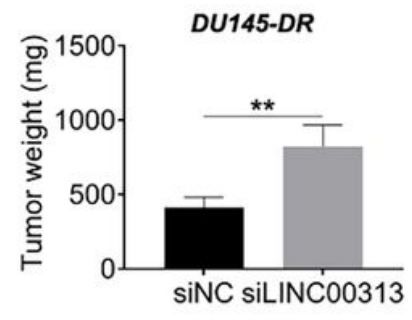

g

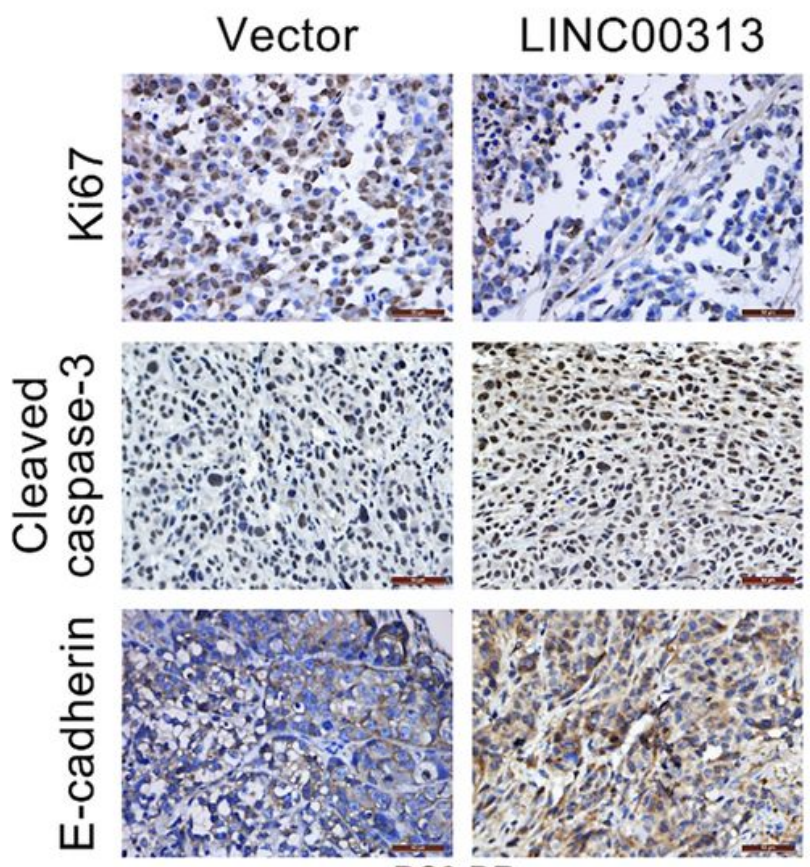

PC3-DR

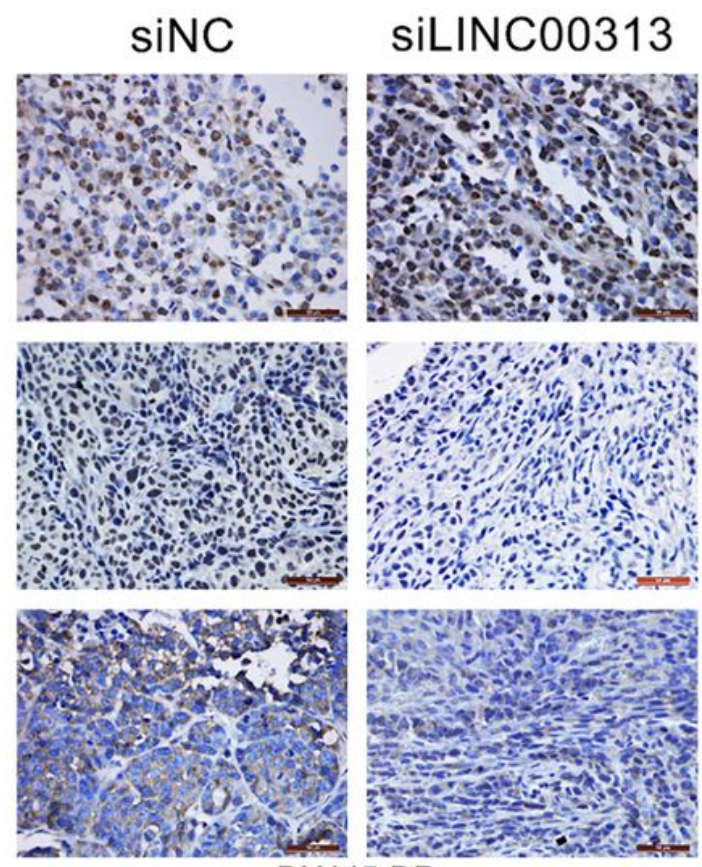

DU145-DR
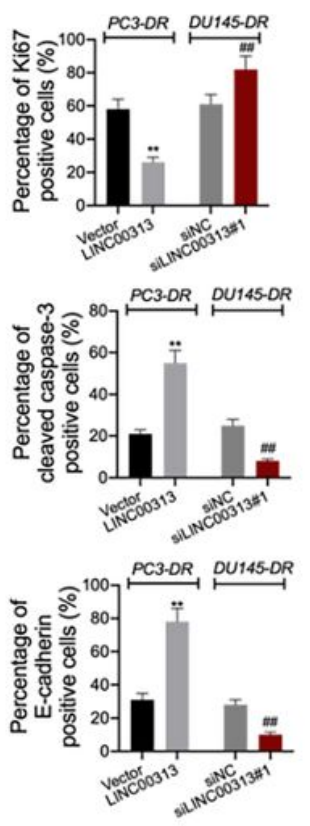

\section{Figure 7}

LINC00313 affected the oncogenicity of dox-resistant PC cells in vivo Vectors expressing LINC00313 transfected PC3-DR cells were subcutaneously injected into the nude mice, (a) The nude mice were sacrificed, and the tumors were collected after 30 day, (b) the volume of the tumors were determined, (c) the weight of the tumors were determined. siRNA targeting LINC00313\#1 transfected DU145-DR cells were subcutaneously injected into the nude mice, $(d)$ The nude mice were sacrificed, and the tumors were collected after 30 day, (e) the volume of the tumors were determined, (f) the weight of the tumors were determined. (g) IHC assay was used to detected Ki67, cleaved caspase-3, and E-cadherin expression in tumor tissue between vector group and LINC00313 group or between siNC group or siLINC00313 group. Data are presented as the mean \pm standard deviation. ${ }^{*} \mathrm{P}<0.01 \mathrm{vs}$. siNC/or vector group. 
a

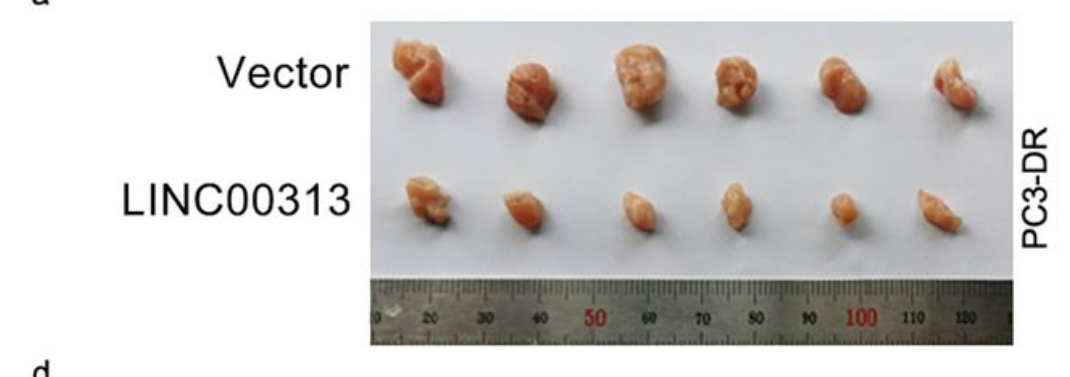

d

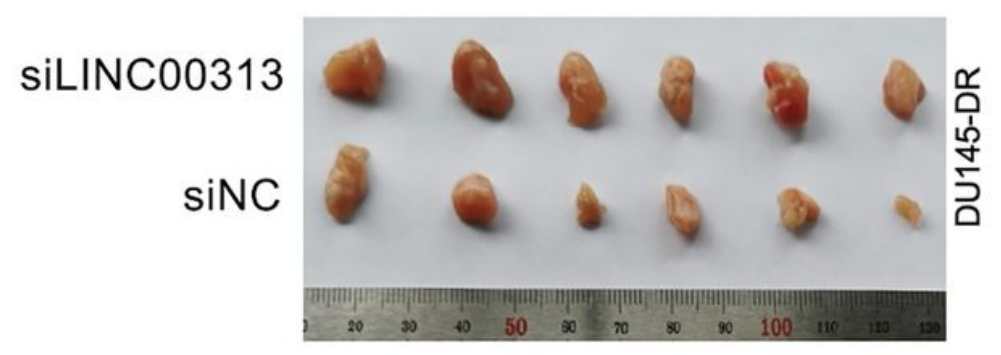

b

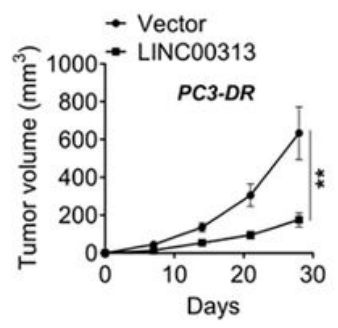

e

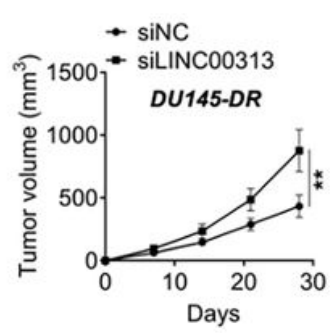

C

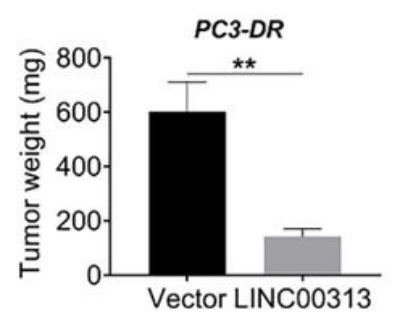

f

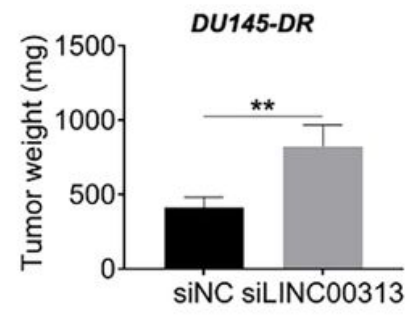

g

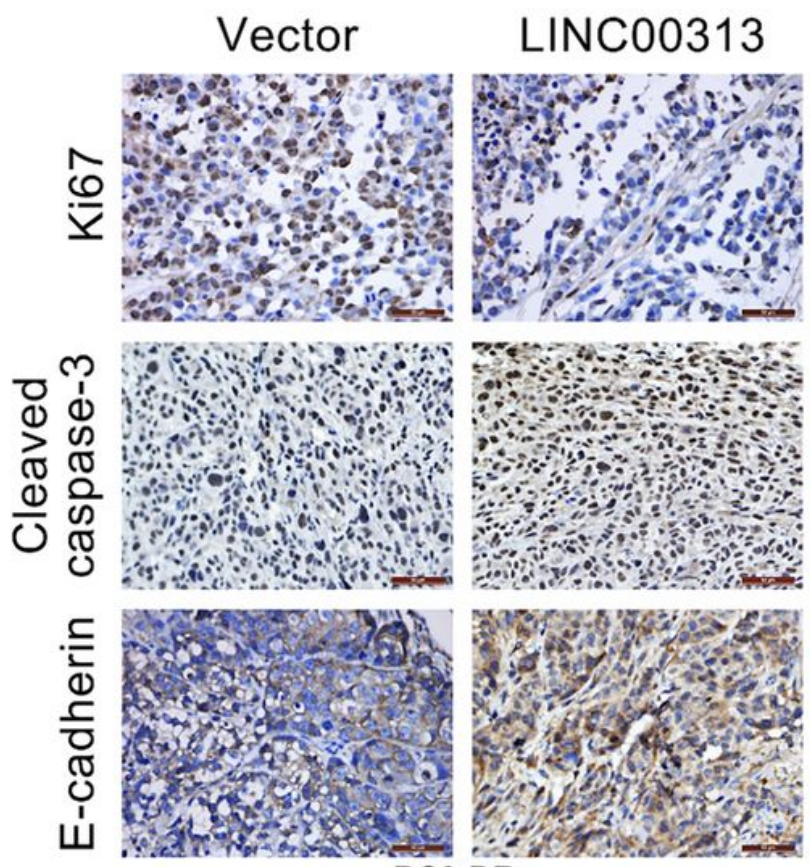

PC3-DR

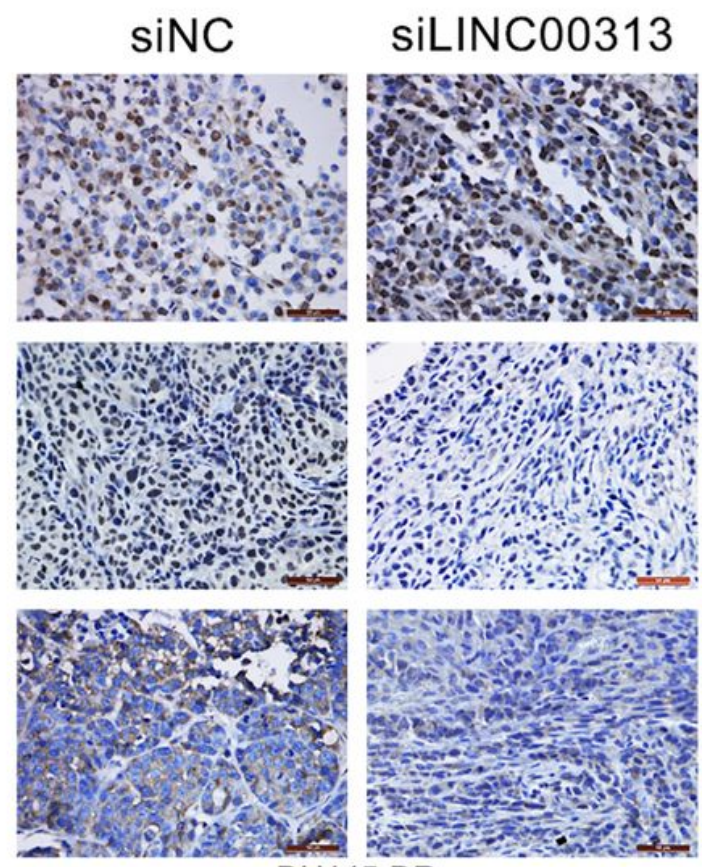

DU145-DR
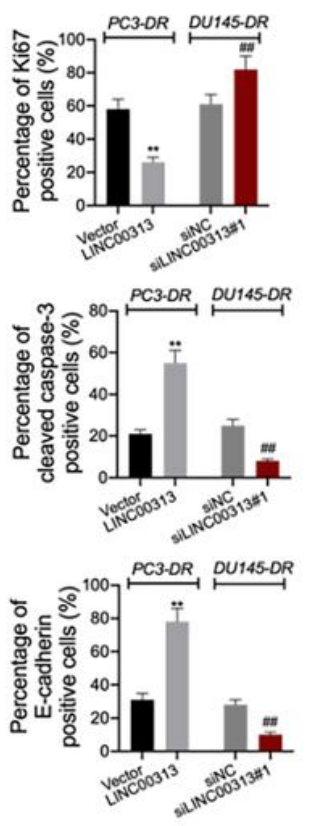

\section{Figure 7}

LINC00313 affected the oncogenicity of dox-resistant PC cells in vivo Vectors expressing LINC00313 transfected PC3-DR cells were subcutaneously injected into the nude mice, (a) The nude mice were sacrificed, and the tumors were collected after 30 day, (b) the volume of the tumors were determined, (c) the weight of the tumors were determined. siRNA targeting LINC00313\#1 transfected DU145-DR cells were subcutaneously injected into the nude mice, $(d)$ The nude mice were sacrificed, and the tumors were collected after 30 day, (e) the volume of the tumors were determined, (f) the weight of the tumors were determined. (g) IHC assay was used to detected Ki67, cleaved caspase-3, and E-cadherin expression in tumor tissue between vector group and LINC00313 group or between siNC group or siLINC00313 group. Data are presented as the mean \pm standard deviation. ${ }^{*} \mathrm{P}<0.01 \mathrm{vs}$. siNC/or vector group. 

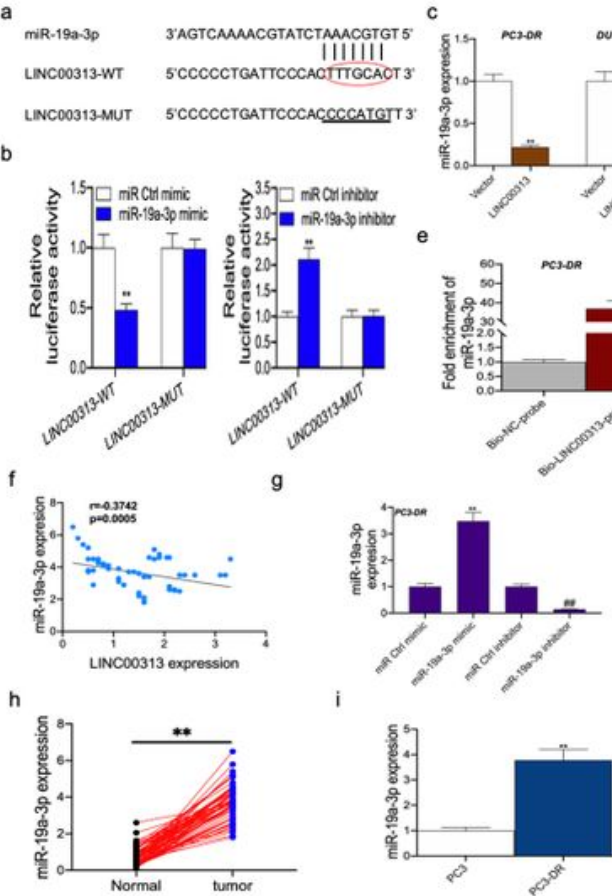

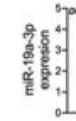
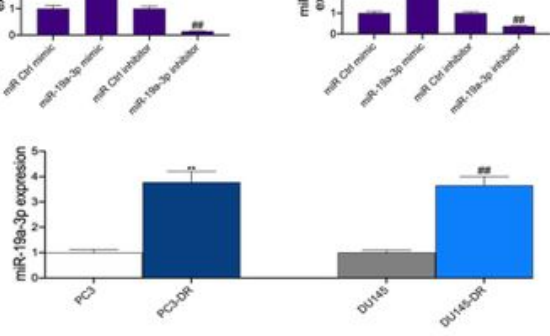

k
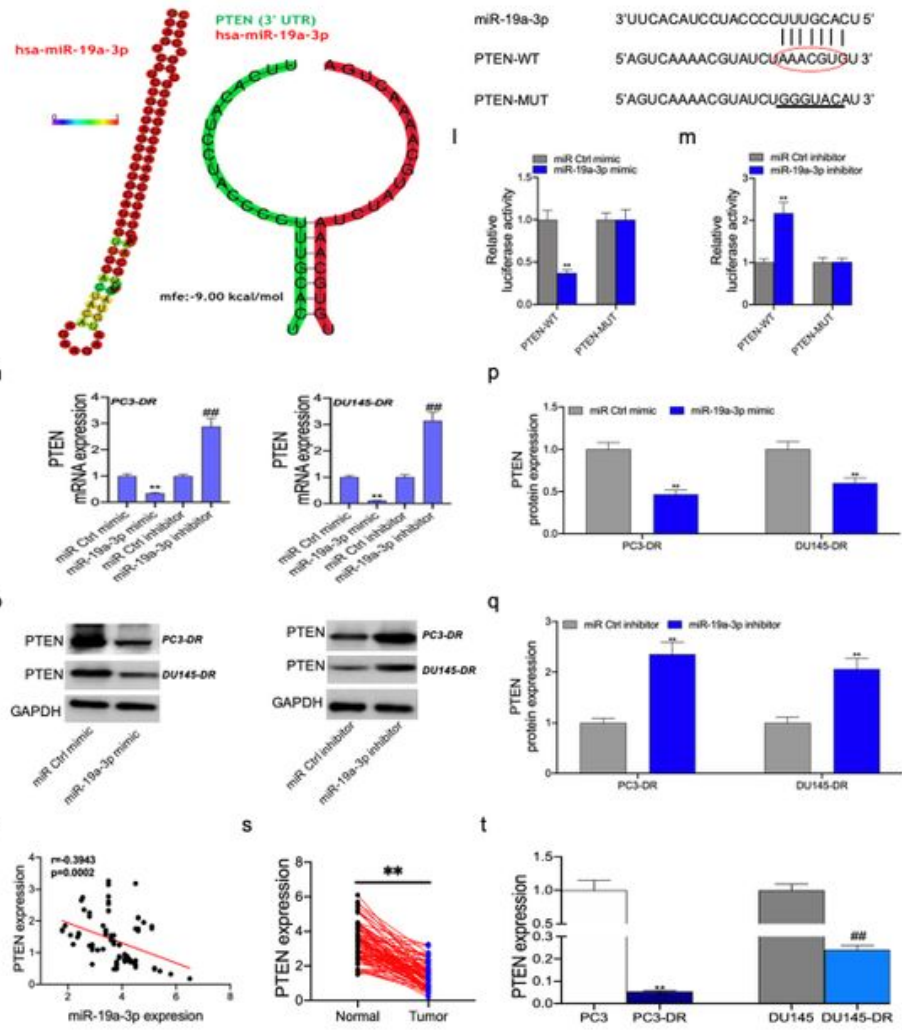

Figure 8

miR-19a-3p, identified as a target miRNA of LINC00313, was upregulated in tumor tissues of patients with PCa and in dox-resistant PCa cells and PTEN was target gene of miR-19a-3p (a) Starbase v2.0 database showed that binding site of LINC00313 and miR-19a-3p; (b) Luciferase reporter assays were used to prove that miR-19a-3p can target LINC00313. After LINC00313 overexpression or inhibition were transfected into PC3-DR, and DU145-DR cells, miR-19a-3p expression in (c) PC3-DR and DU145-DR cells 
was detected by RT-qPCR assay. After siRNA targeting LINC00313\#1 or LINC00313\#2 were transfected into PC3-DR, and DU145-DR cells, miR-19a-3p expression in (d) PC3-DR and DU145-DR cells was detected by RT-qPCR assay. (e) Biotin-labeled LINC00313 RNA and antisense RNA were incubated with PC3-DR, and DU145-DR cells lysate to pull down RNAs, and subsequently RT-PCR was performed to analyze the miR-19a-3p amount. (f) Correlation analysis of LINC00313 and miR-19a-3p. After miR-19a-3p mimic or inhibitor were transfected into PC3-DR, and DU145-DR cells, miR-19a-3p expression in (g) PC3-DR and DU145-DR cells was detected by RT-qPCR assay. (h) The mRNA level of miR-19a-3p between normal adjacent group and PC tumor group was detected by RT-qPCR assay. (i) The mRNA level of miR-19a-3p between PC/or DU145 cells and PC-DR/or DU145-DR cells was detected by RT-qPCR assay. (j) Predicted secondary stem-loop structure of hsa-miR-19a-3p (the colors in each base pair represent its probabilities) and the binding site between miR-19a-3p and PTEN ( $\mathrm{mfe}$ : $-9.00 \mathrm{kcal} / \mathrm{mol}$-generated with BibiServRNAhybrid). (k) A putative binding site of PTEN-3'-UTR for miR-19a-3p was shown. $(\mathrm{l}, \mathrm{m})$ the dual luciferase reporter assays to verify miR-19a-3p targeting PTEN. (n) PTEN mRNA expression in PC3-DR and DU145-DR cells with miR-19a-3p mimic/inhibitor transfection was detected by RT-qPCR. (o-q) PTEN protein expression in PC3-DR and DU145-DR cells with miR-19a-3p mimic/inhibitor transfection was detected by western blotting. ( $r$ ) Correlation analysis of PTEN and miR-19a-3p. (s) The mRNA level of miR-19a-3p between normal adjacent group and PC tumor group was detected by RT-qPCR assay. ( $t$ ) The mRNA level of PTEN between PC/or DU145 cells and PC-DR/or DU145-DR cells was detected by RT-qPCR assay. GAPDH or U6 was used as a load control. Data are presented as the mean \pm standard deviation. $\star \star \mathrm{P}<0.01$ vs. miR Ctrl mimic/or vector/or Bio-NC-probe/or siNC/or tumor/or PC3 group, \#\#P $<0.01$ vs. miR Ctrl inhibitor/or DU145 group. 

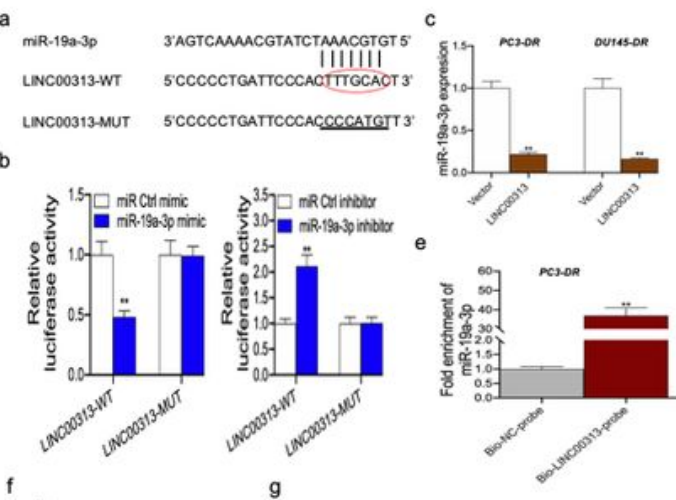

d
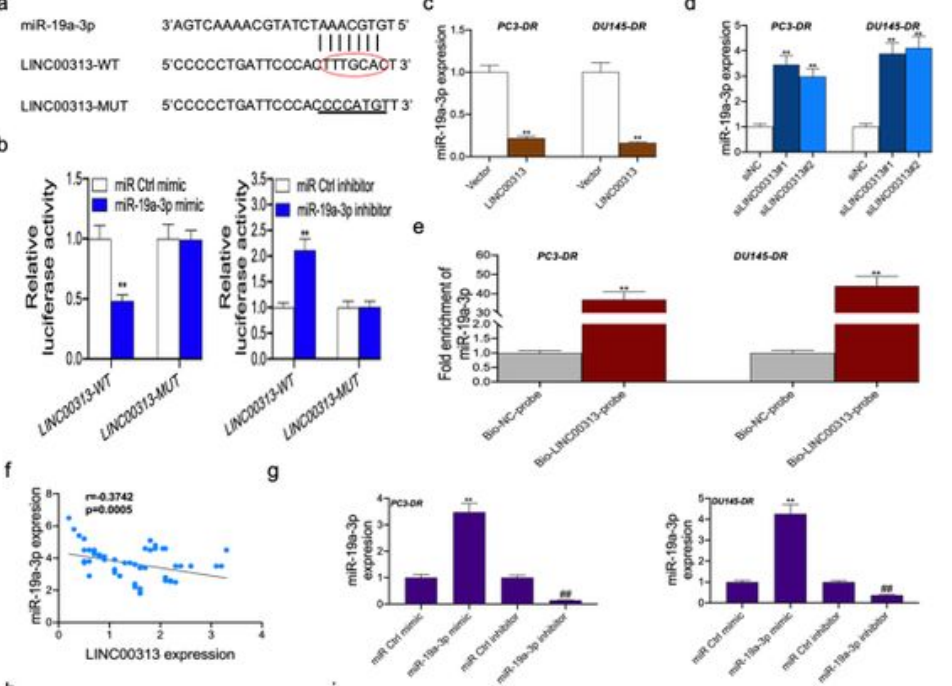

h
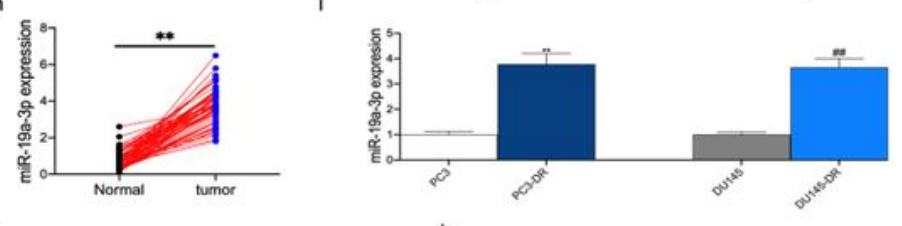

j

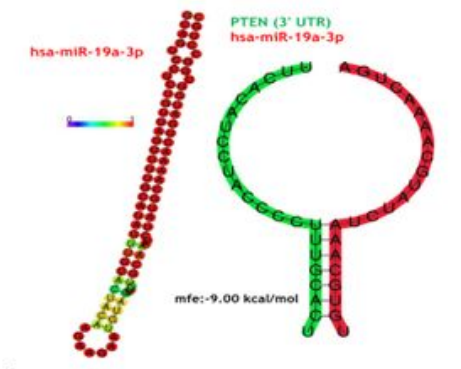

$$
\begin{array}{ll}
\text { MiR-19a-3P } & \text { 3UUCACAUCCUACCCCCUUUGCACUS } \\
\text { PTEN-WT } & \text { SAGUCAAAACGUAUCUAAACGUG III } \\
\text { PTEN-MUT } & \text { 5.AGUCAAAACGUAUCUGGGUACAU } 3
\end{array}
$$

$\mathrm{m}$
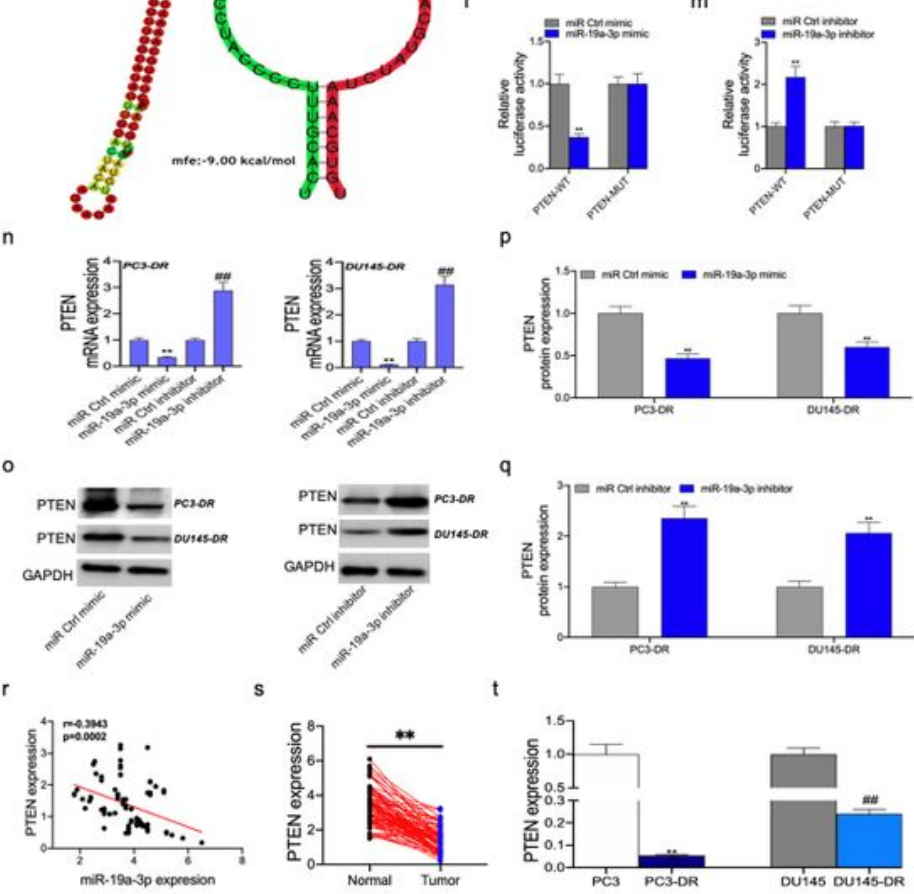

\section{Figure 8}

miR-19a-3p, identified as a target miRNA of LINC00313, was upregulated in tumor tissues of patients with PCa and in dox-resistant PCa cells and PTEN was target gene of miR-19a-3p (a) Starbase v2.0 database showed that binding site of LINC00313 and miR-19a-3p; (b) Luciferase reporter assays were used to prove that miR-19a-3p can target LINC00313. After LINC00313 overexpression or inhibition were transfected into PC3-DR, and DU145-DR cells, miR-19a-3p expression in (c) PC3-DR and DU145-DR cells 
was detected by RT-qPCR assay. After siRNA targeting LINC00313\#1 or LINC00313\#2 were transfected into PC3-DR, and DU145-DR cells, miR-19a-3p expression in (d) PC3-DR and DU145-DR cells was detected by RT-qPCR assay. (e) Biotin-labeled LINC00313 RNA and antisense RNA were incubated with PC3-DR, and DU145-DR cells lysate to pull down RNAs, and subsequently RT-PCR was performed to analyze the miR-19a-3p amount. (f) Correlation analysis of LINC00313 and miR-19a-3p. After miR-19a-3p mimic or inhibitor were transfected into PC3-DR, and DU145-DR cells, miR-19a-3p expression in (g) PC3-DR and DU145-DR cells was detected by RT-qPCR assay. (h) The mRNA level of miR-19a-3p between normal adjacent group and PC tumor group was detected by RT-qPCR assay. (i) The mRNA level of miR-19a-3p between PC/or DU145 cells and PC-DR/or DU145-DR cells was detected by RT-qPCR assay. (j) Predicted secondary stem-loop structure of hsa-miR-19a-3p (the colors in each base pair represent its probabilities) and the binding site between miR-19a-3p and PTEN ( $\mathrm{mfe}$ : $-9.00 \mathrm{kcal} / \mathrm{mol}$-generated with BibiServRNAhybrid). (k) A putative binding site of PTEN-3'-UTR for miR-19a-3p was shown. $(\mathrm{l}, \mathrm{m})$ the dual luciferase reporter assays to verify miR-19a-3p targeting PTEN. (n) PTEN mRNA expression in PC3-DR and DU145-DR cells with miR-19a-3p mimic/inhibitor transfection was detected by RT-qPCR. (o-q) PTEN protein expression in PC3-DR and DU145-DR cells with miR-19a-3p mimic/inhibitor transfection was detected by western blotting. ( $r$ ) Correlation analysis of PTEN and miR-19a-3p. (s) The mRNA level of miR-19a-3p between normal adjacent group and PC tumor group was detected by RT-qPCR assay. ( $t$ ) The mRNA level of PTEN between PC/or DU145 cells and PC-DR/or DU145-DR cells was detected by RT-qPCR assay. GAPDH or U6 was used as a load control. Data are presented as the mean \pm standard deviation. $\star \star \mathrm{P}<0.01$ vs. miR Ctrl mimic/or vector/or Bio-NC-probe/or siNC/or tumor/or PC3 group, \#\#P $<0.01$ vs. miR Ctrl inhibitor/or DU145 group. 
a
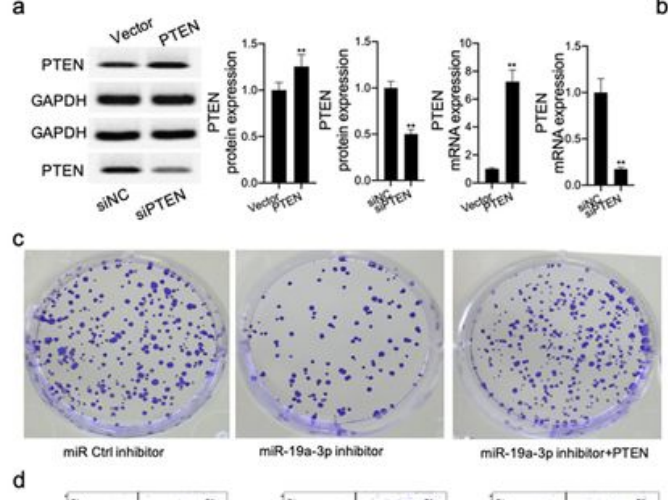

d
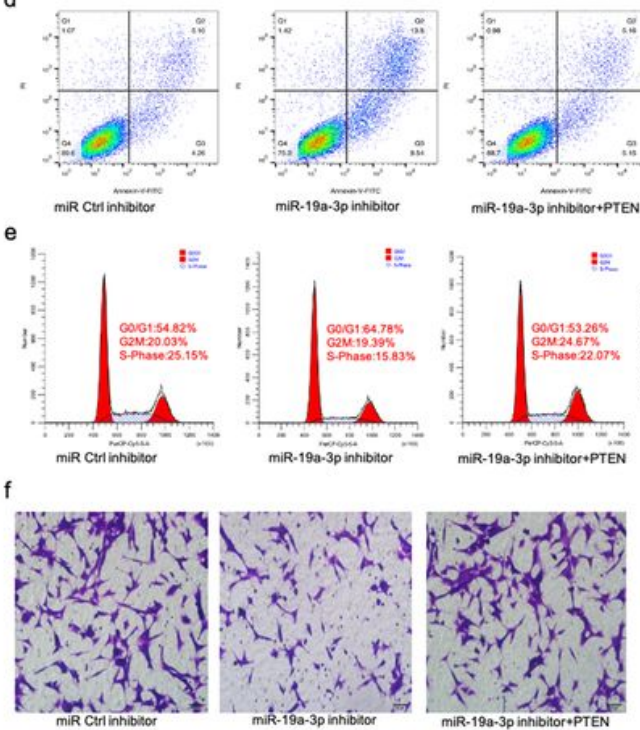

g
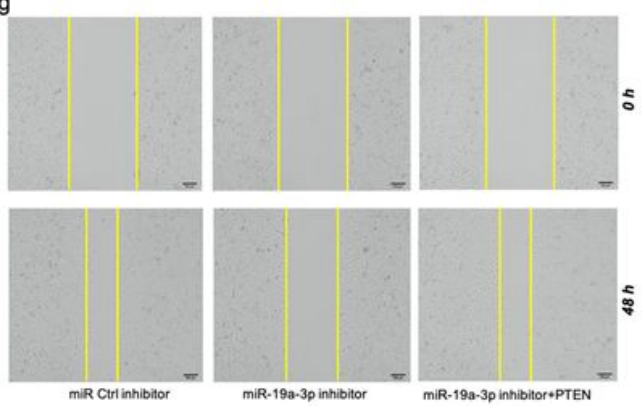
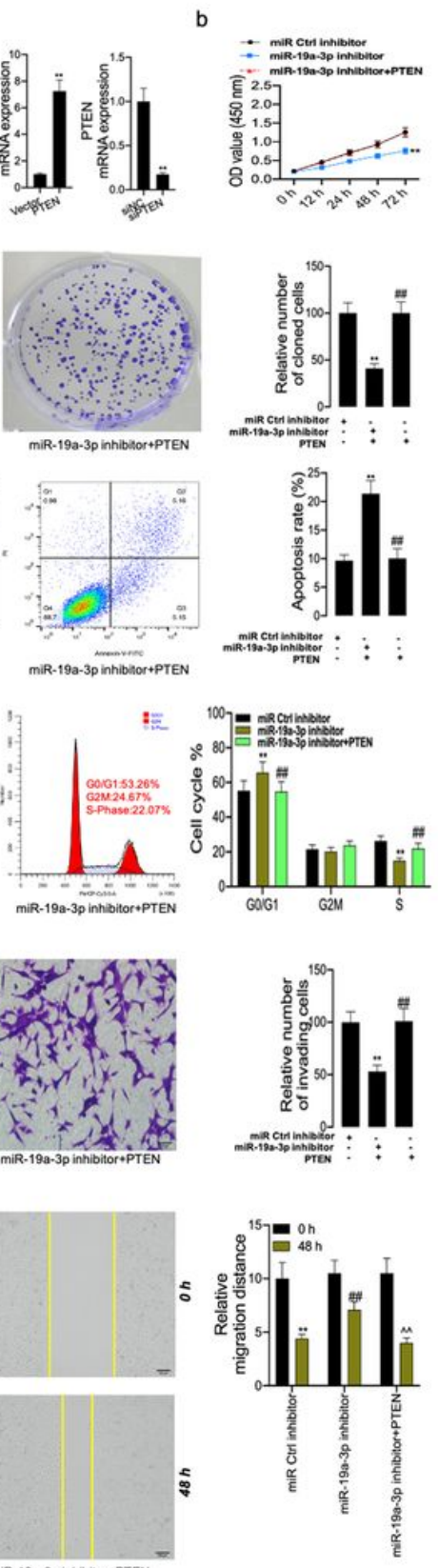

h

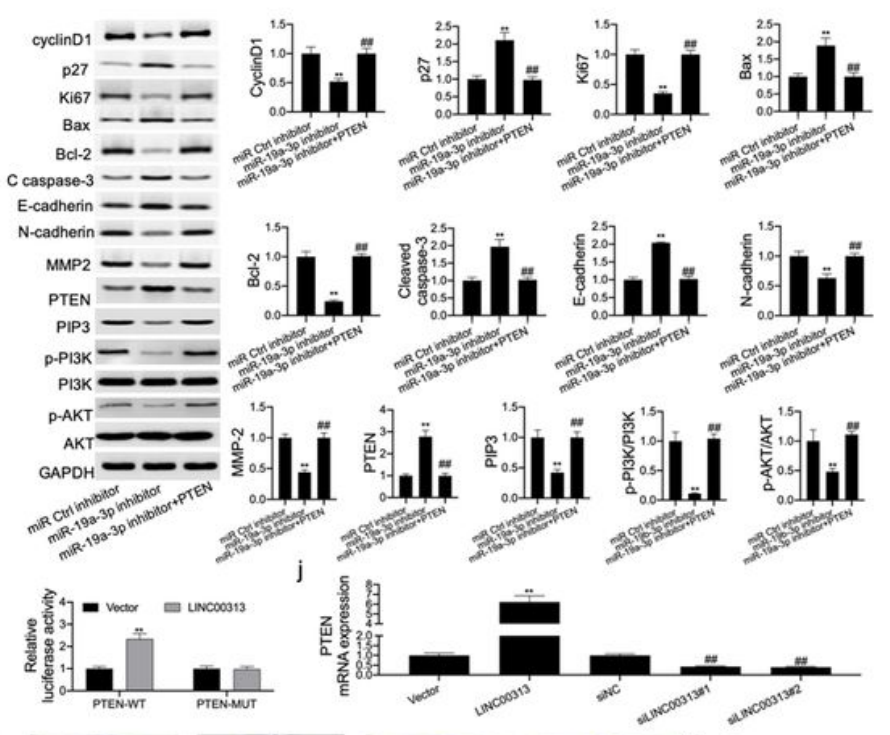

k

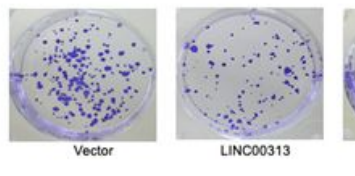

\section{3}
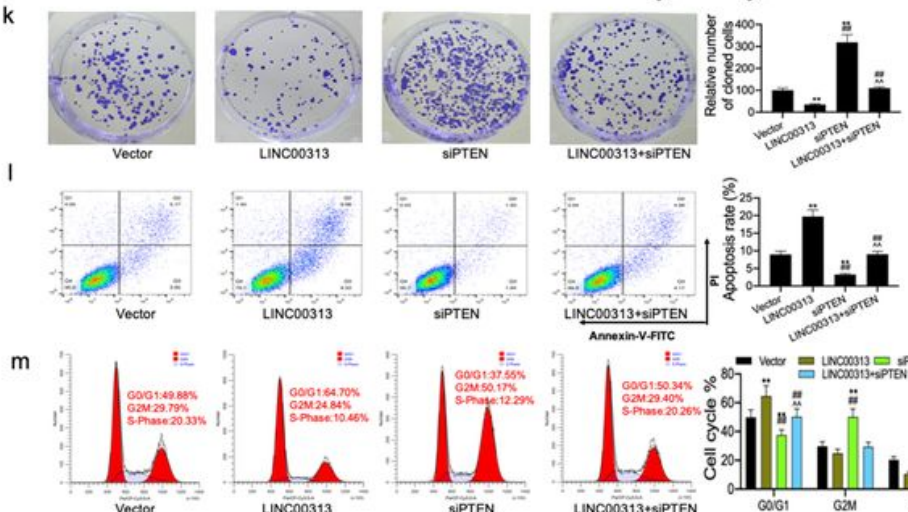

n
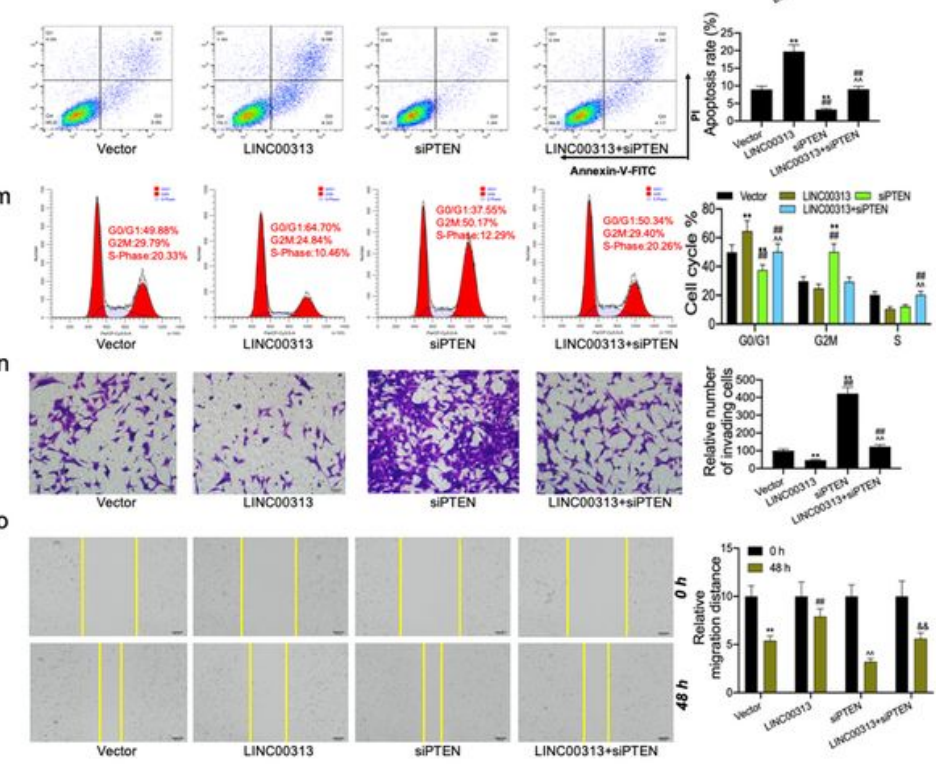

\section{Figure 9}

LINC00313 regulates proliferation, apoptosis, cell cycle, invasion, and migration by targeting miR-19a-3p to affect PTEN expression, thereby regulating the activation of the PI3K/Akt signaling pathway (a) PTEN expression in PTEN and siPTEN groups was detected by RT-qPCR and western blot assay. After miR-19a$3 p$ inhibitor and PTEN overexpression were co-transfected into PC3-DR cells, (b) cell viability was detected by CCK-8 assay, (c) cell proliferation was detected by colony formation assay, (d) cell apoptosis was detected by flow cytometry analysis, (e) cell cycle was detected by flow cytometry analysis, (f) cell invasion was detected by transwell assay, and (g) cell migration was detected by Scratch-Wound assay, and (h) the protein levels of cyclinD1, p27, Ki67, Bax, Bcl-2, cleaved caspase-3/-9, E-cadherin, N-cadherin, Vimentin, MMP-2, PTEN, PIP3, phosph-PI3K, PI3K, phosph-AKT, and AKT were detected by western blot assay. (i) Luciferase reporter assays were used to prove that LINC00313 can target PTEN. (j) PTEN 
expression in LINC00313 and siLINC00313\#1/or \#2 groups was detected by RT-qPCR assay. After LINC00313 overexpression and siPTEN were co-transfected into PC3-DR cells, (k) cell proliferation was detected by colony formation assay, $(\mathrm{l})$ cell apoptosis was detected by flow cytometry analysis, $(\mathrm{m})$ cell cycle was detected by flow cytometry analysis, (n) cell invasion was detected by transwell assay, and (o) cell migration was detected by Scratch-Wound assay. GAPDH or U6 was used as a load control. Data are presented as the mean \pm standard deviation. ${ }^{\star \star P}<0.01$ vs. miR Ctrl inhibitor/or vector/or siNC group, \#\#P $<0.01$ vs. miR-19a-3p inhibitor/or LINC00313 group, and ${ }^{\wedge} \mathrm{P}<0.01$ vs. siPTEN group.

a
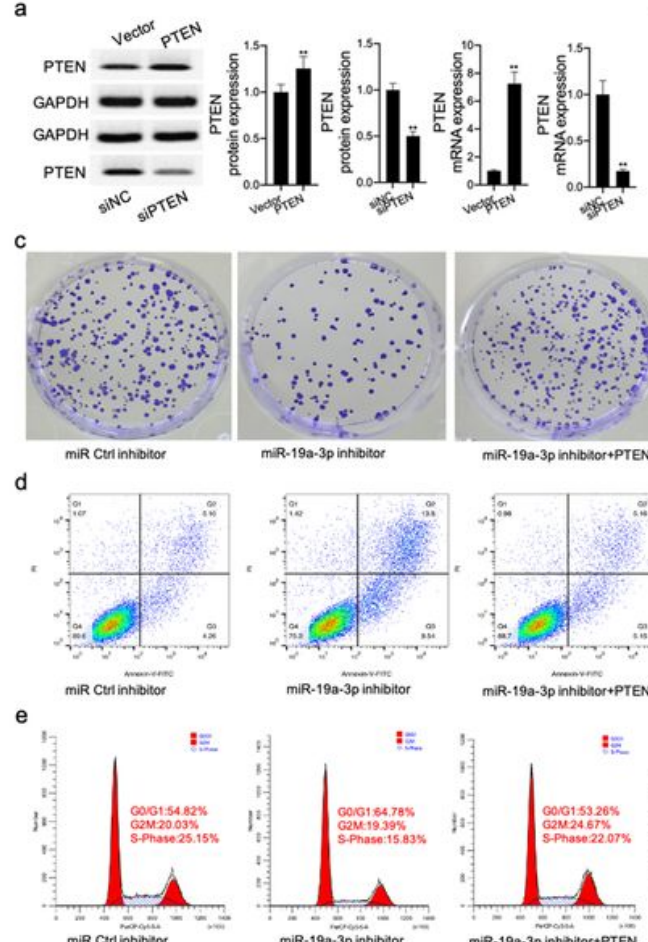

mir Cerrinhibitor
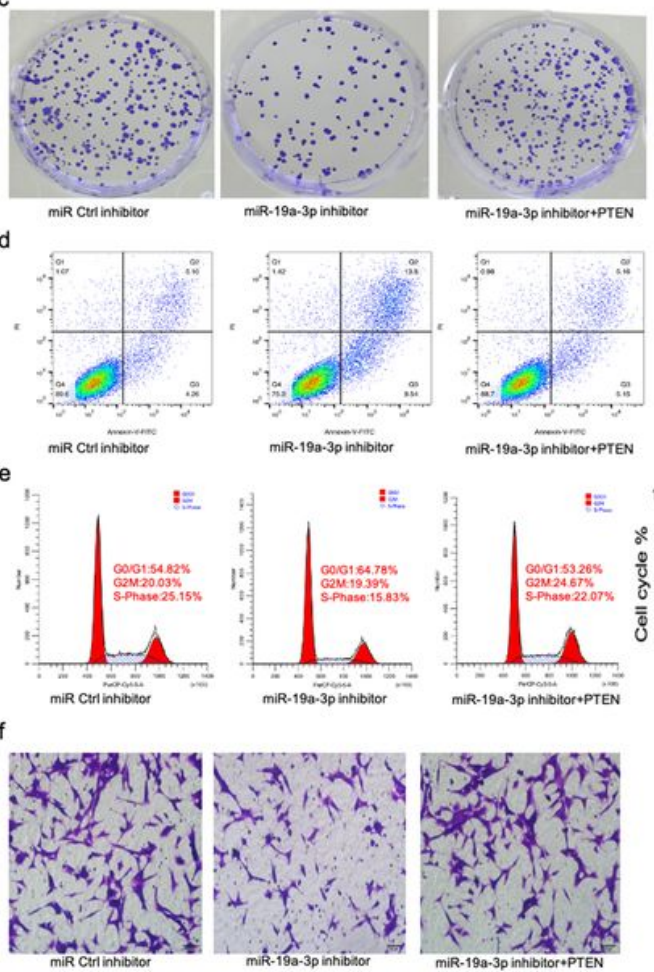

miR-19a-3p inhibitor
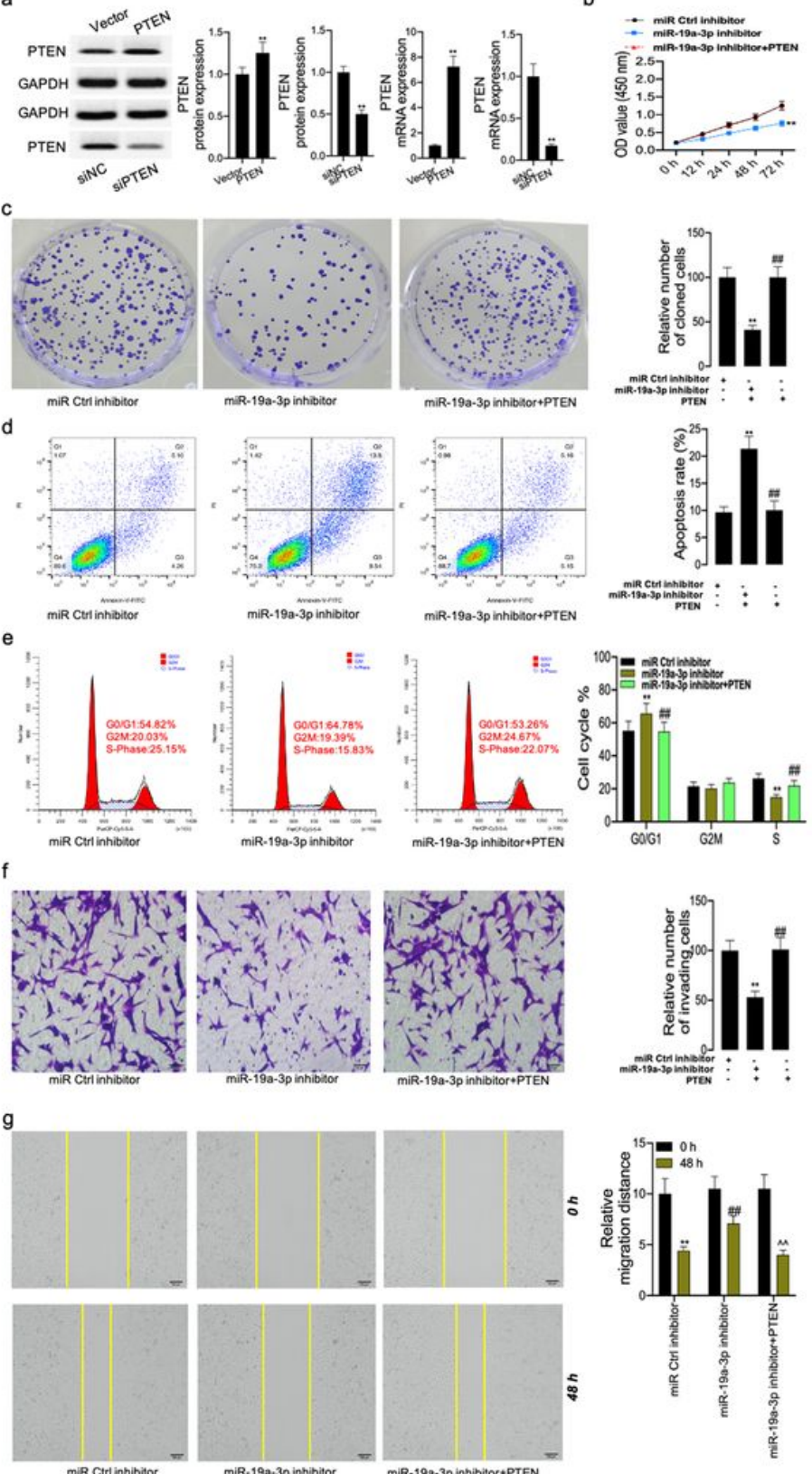
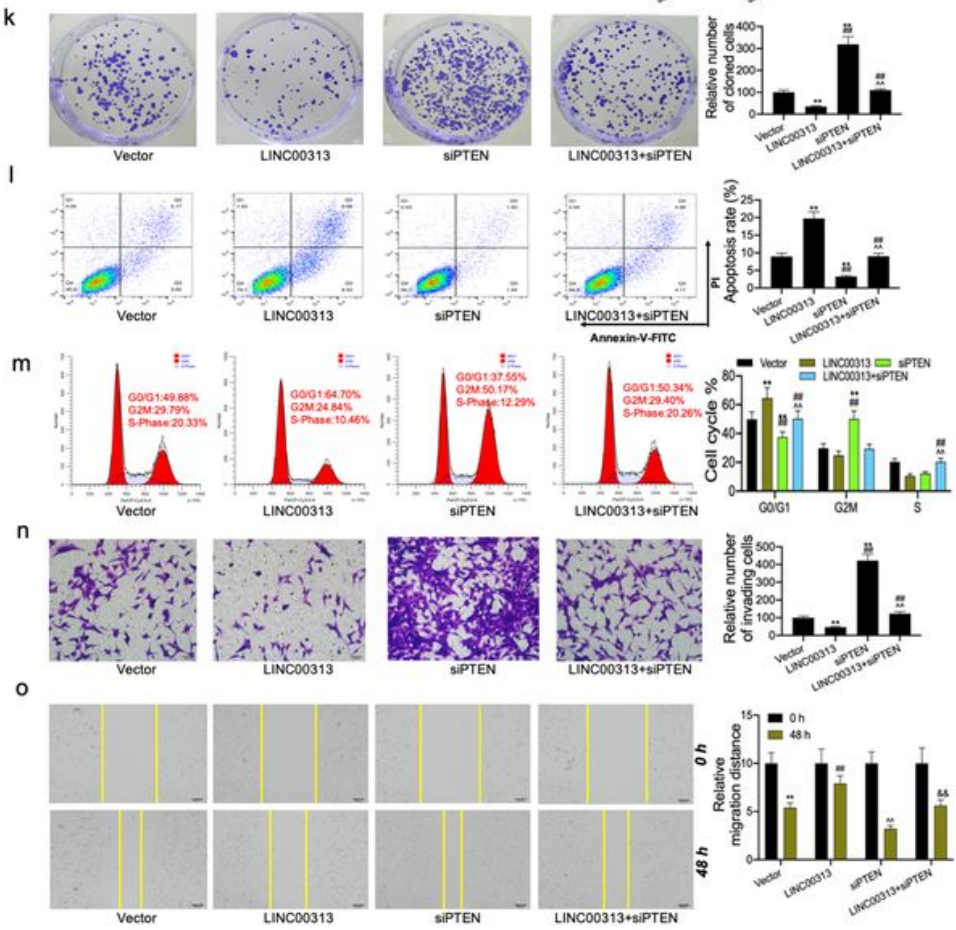

\section{Figure 9}

LINC00313 regulates proliferation, apoptosis, cell cycle, invasion, and migration by targeting miR-19a-3p to affect PTEN expression, thereby regulating the activation of the PI3K/Akt signaling pathway (a) PTEN expression in PTEN and siPTEN groups was detected by RT-qPCR and western blot assay. After miR-19a- 
$3 p$ inhibitor and PTEN overexpression were co-transfected into PC3-DR cells, (b) cell viability was detected by CCK-8 assay, (c) cell proliferation was detected by colony formation assay, (d) cell apoptosis was detected by flow cytometry analysis, (e) cell cycle was detected by flow cytometry analysis, (f) cell invasion was detected by transwell assay, and (g) cell migration was detected by Scratch-Wound assay, and $(\mathrm{h})$ the protein levels of cyclinD1, p27, Ki67, Bax, Bcl-2, cleaved caspase-3/-9, E-cadherin, N-cadherin, Vimentin, MMP-2, PTEN, PIP3, phosph-PI3K, PI3K, phosph-AKT, and AKT were detected by western blot assay. (i) Luciferase reporter assays were used to prove that LINC00313 can target PTEN. (j) PTEN expression in LINC00313 and siLINC00313\#1/or \#2 groups was detected by RT-qPCR assay. After LINC00313 overexpression and siPTEN were co-transfected into PC3-DR cells, (k) cell proliferation was detected by colony formation assay, $(\mathrm{I})$ cell apoptosis was detected by flow cytometry analysis, $(\mathrm{m})$ cell cycle was detected by flow cytometry analysis, ( $n$ ) cell invasion was detected by transwell assay, and (o) cell migration was detected by Scratch-Wound assay. GAPDH or U6 was used as a load control. Data are presented as the mean \pm standard deviation. ${ }^{\star \star} \mathrm{P}<0.01 \mathrm{vs}$. miR Ctrl inhibitor/or vector/or siNC group, \#\#P $<0.01$ vs. miR-19a-3p inhibitor/or LINC00313 group, and ${ }^{\wedge}{ }^{\wedge} \mathrm{P}<0.01$ vs. SiPTEN group. 